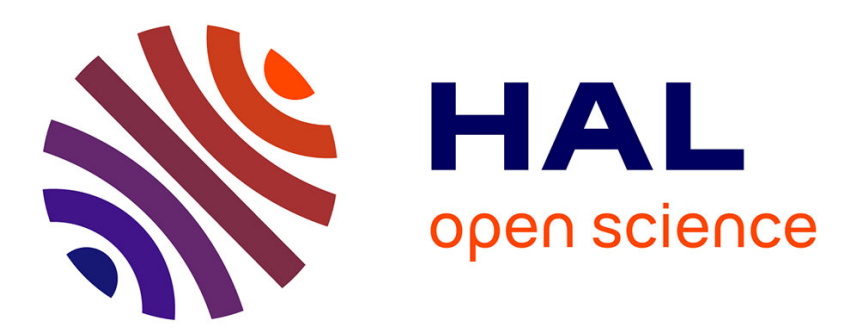

\title{
Nonlinear optimal perturbations in a Couette flow: bursting and transition
}

Stefania Cherubini, Pietro de Palma

\section{To cite this version:}

Stefania Cherubini, Pietro de Palma. Nonlinear optimal perturbations in a Couette flow: bursting and transition. Journal of Fluid Mechanics, 2013, 716, pp.251 -279. 10.1017/jfm.2012.544 . hal-00799751

\section{HAL Id: hal-00799751 \\ https://hal.science/hal-00799751}

Submitted on 12 Mar 2014

HAL is a multi-disciplinary open access archive for the deposit and dissemination of scientific research documents, whether they are published or not. The documents may come from teaching and research institutions in France or abroad, or from public or private research centers.
L'archive ouverte pluridisciplinaire HAL, est destinée au dépôt et à la diffusion de documents scientifiques de niveau recherche, publiés ou non, émanant des établissements d'enseignement et de recherche français ou étrangers, des laboratoires publics ou privés. 


\title{
Nonlinear optimal perturbations in a Couette flow: bursting and transition
}

\author{
S. Cherubini ${ }^{1,2, \dagger}$ and P. De Palma ${ }^{2}$ \\ ${ }^{1}$ DynFluid, Arts et Metiers ParisTech, 151, boulevard de l'Hopital, 75013 Paris, France \\ ${ }^{2}$ DIMeG, CEMeC, Politecnico di Bari, via Re David 200, 70125 Bari, Italy \\ (Received 7 December 2011; revised 4 September 2012; accepted 5 November 2012)
}

This paper provides the analysis of bursting and transition to turbulence in a Couette flow, based on the growth of nonlinear optimal disturbances. We use a global variational procedure to identify such optimal disturbances, defined as those initial perturbations yielding the largest energy growth at a given target time, for given Reynolds number and initial energy. The nonlinear optimal disturbances are found to be characterized by a basic structure, composed of inclined streamwise vortices along localized regions of low and high momentum. This basic structure closely recalls that found in boundary-layer flow (Cherubini et al., J. Fluid Mech., vol. 689, 2011, pp. 221-253), indicating that this structure may be considered the most 'energetic' one at short target times. However, small differences in the shape of these optimal perturbations, due to different levels of the initial energy or target time assigned in the optimization process, may produce remarkable differences in their evolution towards turbulence. In particular, direct numerical simulations have shown that optimal disturbances obtained for large initial energies and target times induce bursting events, whereas for lower values of these parameters the flow is directly attracted towards the turbulent state. For this reason, the optimal disturbances have been classified into two classes, the highly dissipative and the short-path perturbations. Both classes lead the flow to turbulence, skipping the phases of streak formation and secondary instability which are typical of the classical transition scenario for shear flows. The dynamics of this transition scenario exploits three main features of the nonlinear optimal disturbances: (i) the large initial value of the streamwise velocity component; (ii) the streamwise dependence of the disturbance; (iii) the presence of initial inclined streamwise vortices. The short-path perturbations are found to spend a considerable amount of time in the vicinity of the edge state (Schneider et al., Phys. Rev. E, vol. $78,2008,037301$ ), whereas the highly dissipative optimal disturbances pass closer to the edge, but they are rapidly repelled away from it, leading the flow to high values of the dissipation rate. After this dissipation peak, the trajectories do not lead towards the turbulent attractor, but they spend some time in the vicinity of an unstable periodic orbit (UPO). This behaviour led us to conjecture that bursting events can be obtained not only as homoclinic orbits approaching the UPO, as recently found by van Veen \& Kawahara (Phys. Rev. Lett., vol. 107, 2011, p. 114501), but also as heteroclinic orbits between the equilibrium solution on the edge and the UPO.

Key words: nonlinear dynamical systems, nonlinear instability, transition to turbulence 


\section{Introduction}

Despite the important achievements in the last twenty years, flow instability and transition to turbulence remain challenging fields of research due to the richness of the basic phenomena involved. In fact, also for very simple shear flows, such as pipe flows or Couette flows, the mechanisms of laminar-turbulent transition are formidably complex. For such flows, small perturbation theory predicts stability of the laminar profile for $R e \rightarrow \infty$ (Re being the Reynolds number), but transition is observed already at small values of $R e$. In recent years, some light has been shed on this apparently strange behaviour thanks to the development of a new dynamical system approach to turbulence. Many authors have assumed that turbulence in these flows is linked to the existence (for values of $R e$ larger than a certain threshold) of exact coherent states (ECS), which are either steady, or time-periodic, or chaotic solutions of the Navier-Stokes equations, containing the basic flow structures which can be considered the kernel of a turbulent flow; see, for instance, Nagata (1990, 1997), Waleffe (1998), Faisst \& Eckhardt (2003), Wedin \& Kerswell (2004) and Gibson, Halcrow \& Cvitanović (2009). Being unstable, such coherent states cannot be directly observed in experiments and simulations, although the flow can 'visit' them, its trajectory in phase space being strongly deviated in their vicinity; see Hof et al. (2004) and Eckhardt et al. (2007). These states are fundamental for the flow dynamics, since the entanglement of their stable and unstable manifolds is at the basis of the chaotic walk of the system's trajectory in phase space towards the turbulent state. In particular, the upper branch solutions are found to capture well the turbulent dynamics (Jimenez et al. 2005), whereas the lower branch models the transitional dynamics (Waleffe 2003). A systematic assessment of the fact that lower branch solutions are embedded in the flow dynamics during transition was provided by Kerswell \& Tutty (2007) for pipe flow, demonstrating the relevance of such solutions in the transition scenario, as suggested before by Itano \& Toh (2001) for plane Poiseuille flow.

For Couette flow, the lower branch solution has only one unstable direction (Wang, Gibson \& Waleffe 2007), so that its stable manifold divides the phase space into two subspaces: the basin of attraction of the laminar flow, and the ensemble of states which evolve towards turbulence. In this particular case, the laminar-turbulent boundary (the edge of turbulence: see Skufca, Yorke \& Eckhardt 2006) coincides with the stable manifold of the lower branch solution, and the edge state, which is the relative attractor lying on the laminar-turbulent boundary, is the saddle state itself. Being a saddle in phase space, the edge state for a Couette flow is one of the key states for controlling transition to turbulence. Thus, it is fundamental to determining which kind of perturbation is able to efficiently bring the flow trajectory towards the edge of turbulence.

Following these ideas, Monokrousos et al. (2011) very recently computed the initial condition on the laminar-turbulent boundary which represents the closest state to the laminar solution for a plane Couette flow. Using non-equilibrium theory they found the perturbation of minimal initial energy which reaches a statistically steady turbulent state at a large time. To this end, they optimized the time-averaged dissipation for different values of the initial energy and bisected such values until they found the threshold value of the initial energy for which a turbulent state can be established at large times. This approach is very useful for optimizing the route that leads directly to the statistically steady turbulent state; on the other hand, being based on time-averaged quantities, it cannot capture strong transient behaviours of the flow such as bursting events, which are an important feature of the intermittent nature of turbulence. 
Bursting is usually linked to the passage of coherent structures in the near-wall regions, which transfer energy from the large to the small scales, producing turbulent fluctuations (Kline et al. 1967). Thus, bursting processes are found to be responsible for the production of a large amount of turbulent kinetic energy in shear flows (for a review see Robinson 1991). Moreover, they are interesting because they provide an example of spatial and temporal intermittency, which are typical of the subcritical transition to turbulence in shear flows. The near-wall flow structures observed during such processes are very different from those associated with the regeneration cycle that sustains turbulence (Waleffe 1997), indicating that the basic mechanisms are different from that inducing a statistically steady turbulent state (for instance, see Itano \& Toh 2001). Very recently, van Veen \& Kawahara (2011) have shown that, for a Couette flow, bursting events are linked to homoclinic orbits approaching an unstable periodic orbit. Along these homoclinic orbits, the vortical structures and the associated energy dissipation are localized in the near-wall region, producing a flow dynamics very different from the regeneration cycle theorized by Waleffe (1997). However, it is still not clear whether - and how - it is possible to efficiently induce the bursting phenomenon starting from the laminar solution. It can thus be interesting to investigate which initial conditions are able to efficiently induce bursting events, and compare them with those that lead directly towards the turbulent attractor.

Therefore, in this paper we compute optimal perturbations which are able to induce high-energy transients in the flow at short times. To this end, we use a Lagrange multiplier optimization in a nonlinear framework, looking for the perturbations of the laminar base flow which are able to induce the largest perturbation energy growth at a short target time. Very recently, the nonlinear optimizations performed by Pringle \& Kerswell (2010), Cherubini et al. (2010a), Monokrousos et al. (2011) and Cherubini et al. (2011) have provided a breakthrough on the importance of nonlinearity on the amplification mechanisms leading to turbulence. These investigations have shown the existence of a preferential route to transition, initiated by localized disturbances of finite amplitude, grounded on nonlinear effects. For pipe flow, the mechanism seems that of the oblique transition (Pringle \& Kerswell 2010), whereas for boundarylayer flow, it is based on the initial inclination of the vortices inducing defects and dislocations within the flow (Cherubini et al. 2011). On the other hand, for Couette flow, it is still not clear on which mechanisms such a nonlinear preferential route to transition is based, and whether these mechanisms depend on the parameters of the optimization such as the initial energy, the target time and the domain size.

Thus, the aim of this work is to analyse in detail the mechanisms leading the nonlinear optimal perturbations to bursting and transition, also by investigating the influence on such perturbations of the exact coherent states populating phase space for Couette flow. The perturbations inducing the largest energy growth at short times in Couette flow are computed for several initial energies and target times, and for two different computational domains. For target times and initial energies larger than a threshold value, the optimal perturbation is found to be characterized by a fundamental basic structure, which is formed by a localized array of vortices inclined with respect to the streamwise direction, together with localized high- and low-momentum regions. This structure is capable of maximizing the energy growth at short times, inducing strong peaks of the perturbation energy and dissipation rate (bursting events). Direct numerical simulations have been employed to study the mechanism of transition to turbulence and bursting of these nonlinear optimal disturbances. Finally, the role of the exact coherent states, such as those found by Schneider et al. (2008) and Kawahara \& Kida (2001), respectively, on the establishment of a bursting event, is investigated. 
The paper is organized as follows. In $\S 2$ we define the problem and describe the nonlinear optimization method. In $\S 3$, a thorough discussion of the results of the nonlinear optimization analysis is provided. In particular, in $\S 3.1$, the focus is on the characterization of the optimal perturbation, whereas $\S 3.2$ deals with the optimal route to turbulence, and with the ECS role in this scenario of transition. Finally, in $\S 4$, concluding remarks are provided.

\section{Problem formulation}

\subsection{Governing equations and numerical method}

The behaviour of an incompressible flow is governed by the Navier-Stokes (NS) equations:

$$
\begin{gathered}
\boldsymbol{u}_{t}+(\boldsymbol{u} \cdot \nabla) \boldsymbol{u}=-\nabla p+\frac{1}{R e} \nabla^{2} \boldsymbol{u}, \\
\nabla \cdot \boldsymbol{u}=0,
\end{gathered}
$$

where $\boldsymbol{u}$ is the velocity vector and $p$ is the pressure term (including the contribution of conservative-force fields). For the case of Couette flow considered here, the upper and lower plates are placed at $y= \pm h$, and they move in the streamwise direction at constant velocities $\pm U_{w}$, respectively. Dimensionless variables are defined with respect to half of the velocity difference between the two walls, $U_{w}$, and half of the distance between the plates, $h$, so that the Reynolds number is $R e=U_{w} h / v, v$ being the kinematic viscosity. Two computational domains have been employed. The reference one (hereafter called D1) has dimensions equal to $L_{x}=4 \pi, L_{y}=2$ and $L_{z}=2 \pi, x, y$ and $z$ being the streamwise, wall-normal and spanwise directions, respectively. It is the same domain used in Schneider et al. (2008) for the computation of the edge state, and in Monokrousos et al. (2011) for the computation of the minimal perturbation reaching the edge of turbulence. The second domain (D2) has dimensions $L_{x}=8 \pi$, $L_{y}=2$ and $L_{z}=4 \pi$. At the walls, the no-slip boundary condition is prescribed, whereas in the spanwise and streamwise direction periodicity is imposed for the three velocity components. The NS equations are discretized by a finite-difference fractional-step method with second-order accuracy in time (Verzicco \& Orlandi 1996). A second-order-accurate centred space discretization is used on a staggered grid. After a grid-convergence analysis, we have selected a mesh made up of $201 \times 100 \times 61$ points for D1 and $401 \times 100 \times 121$ points for D2, both clustered towards the walls so that the thickness of the first cell close to the walls is equal to 0.015 . The code has been validated by computing, via an edge-tracking procedure, the steady lower branch solution of Schneider et al. (2008). In particular, the value of the energy density on the edge and the shape of the steady solution have been verified.

\subsection{Nonlinear optimization}

For the optimization procedure, the nonlinear behaviour of a perturbation $\boldsymbol{q}=$ $\left(u^{\prime}, v^{\prime}, w^{\prime}, p^{\prime}\right)^{\mathrm{T}}$ evolving in the Couette flow is studied by employing the NS equations written in a perturbative formulation, with respect to the exact laminar solution, $\boldsymbol{Q}=(U, 0,0, P)^{\mathrm{T}}$, namely, the base flow. In order to find the perturbation at $t=0$ providing the largest disturbance growth at a given target time, $T$, a Lagrange multiplier technique is used (Zuccher, Luchini \& Bottaro 2004; Pringle \& Kerswell 2010). The disturbance energy density is defined as $E(t)=\left\langle\boldsymbol{u}^{\prime}(t), \boldsymbol{u}^{\prime}(t)\right\rangle$, 
where $\boldsymbol{u}^{\prime}=\left(u^{\prime}, v^{\prime}, w^{\prime}\right)$ and the brackets denote the scalar product

$$
\langle\boldsymbol{v}, \boldsymbol{u}\rangle=\frac{V_{r e f}}{V} \int_{V} \boldsymbol{v}^{\mathrm{T}} \boldsymbol{u} \mathrm{d} V .
$$

It is noteworthy that, for purposes of comparison between the domains D1 and D2, the scalar product is scaled by the factor $V_{\text {ref }} / V$, where $V_{\text {ref }}=4 \pi \times 2 \times 2 \pi$ is the volume of the reference domain D1. In the following, $E(t)$ will be simply called energy, for brevity. The aim is to find the initial perturbation $\boldsymbol{q}_{0}$ of given initial energy $E(0)=E_{0}$ which can induce at target time $T$ the largest energy $E(T)$. Thus, the objective function of the procedure, $\mathfrak{I}$, is the energy gain $\mathfrak{s}=E(T) / E(0)$. The Lagrange multiplier technique consists in searching for extrema of an augmented functional, $\mathscr{L}$, with respect to every independent variable, where $\mathscr{L}$ is defined by

$$
\begin{aligned}
\mathscr{L}= & \frac{E(T)}{E(0)}-\int_{0}^{T}\left\langle\boldsymbol{u}^{\dagger},\left\{\frac{\partial \boldsymbol{u}^{\prime}}{\partial t}+\boldsymbol{u}^{\prime} \cdot \nabla \boldsymbol{U}+\boldsymbol{U} \cdot \nabla \boldsymbol{u}^{\prime}+\boldsymbol{u}^{\prime} \cdot \nabla \boldsymbol{u}^{\prime}+\nabla p^{\prime}-\frac{\nabla^{2} \boldsymbol{u}^{\prime}}{R e}\right\}\right\rangle \mathrm{d} t \\
& -\int_{0}^{T}\left\langle p^{\dagger}, \nabla \cdot \boldsymbol{u}^{\prime}\right\rangle \mathrm{d} t-\lambda\left(\frac{E_{0}}{E(0)}-1\right) .
\end{aligned}
$$

The three-dimensional incompressible NS equations in a perturbative formulation (direct equations) and the value of the initial energy are imposed as constraints, using the Lagrange multipliers $\boldsymbol{q}^{\dagger}=\left(\boldsymbol{u}^{\dagger}, p^{\dagger}\right)$, which are defined as the adjoint variables. Integrating by parts and setting to zero the first variation of $\mathscr{L}$ with respect to $u^{\prime}, v^{\prime}, w^{\prime}, p^{\prime}$ leads to the adjoint equations, which are linked to the direct ones by the presence of direct variables in the advection terms. The direct and adjoint equations are parabolic in the forward and backward time direction, respectively, so that they can be solved by a coupled iterative approach. By solving the direct and adjoint equations at each step of the iterative procedure, the first variation of the augmented functional with respect to $\boldsymbol{q}$ and $\boldsymbol{q}^{\dagger}$ is set to zero. Moreover, the gradient of $\mathscr{L}$ with respect to the initial state $\boldsymbol{q}_{0}$ is forced to vanish by means of a conjugate gradient algorithm. Each iteration requires the integration of the three-dimensional direct and adjoint equations forward and backward in time up to the target time. Due to the presence of the direct variables in the advection terms of the adjoint equations, the whole direct-variable field needs to be stored at each time step, requiring a remarkable storage capacity. Moreover, due to stability issues of the time-discretization method, the time step of the direct and adjoint computations should be rather low, the minimum one used here being equal to 0.001 . Thus, the computational effort required to optimize the solution for a single set of the independent parameters $\left(T, R e, E_{0}\right)$ is very high. The whole method is better detailed in Cherubini et al. (2011).

An example of the convergence history of the method for the case with $T=50, E_{0}=0.005$, and $R e=400$ is provided in figure $1(a)$. This figure shows the residual, $r=1 / V\left\langle\partial \mathscr{L} / \partial \boldsymbol{u}^{\prime}, \partial \mathscr{L} / \partial \boldsymbol{u}^{\prime}\right\rangle$; the error of the objective function, $e=$ $\left(E(T)^{n}-E(T)^{n-1}\right) / E(T)^{n}$; and the value of the energy gain. The residual reduces to $10^{-12}$ in $\sim 400$ iterations; then, it decreases very slowly, showing a plateau up to $n=1000$, without any jump or oscillating behaviour such as those found in Pringle, Willis \& Kerswell (2012) for pipe flow. This smooth behaviour is probably due to the short target times and the low Reynolds number considered here. For larger initial energies, the convergence history shows a similar smooth trend, but the residual curve asymptotically levels off at larger values, probably due to the onset of unsteadiness in the solution at target time. This is shown in figure $1(b, c)$ for the case with $T=30$, $R e=400$, with the two initial energies, $E_{0}=0.025$ and $E_{0}=0.1$, respectively. On 

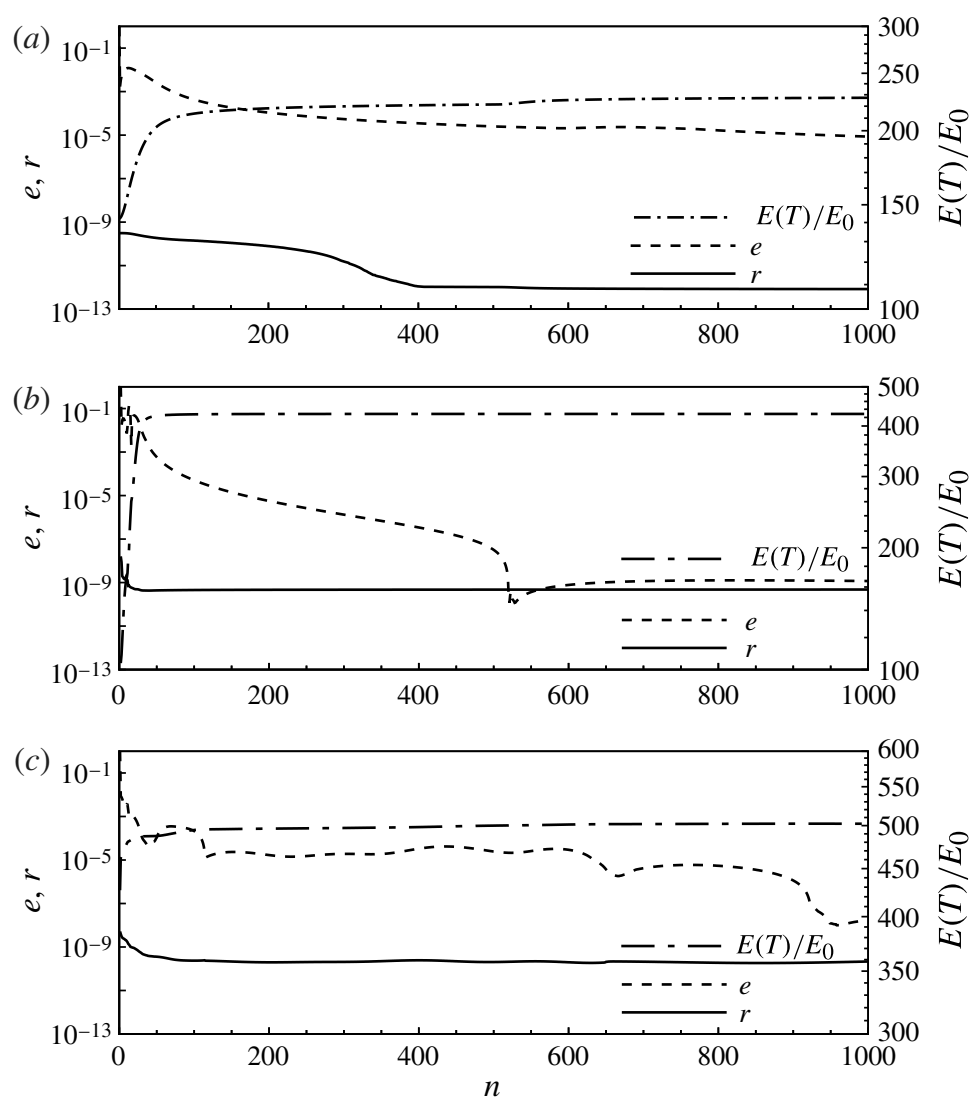

FIgURE 1. Convergence history for a nonlinear optimization at target time $(a) T=50$ with $E_{0}=0.005$, (b) $T=30$ with $E_{0}=0.025$, and $(c) T=30$ with $E_{0}=0.1$. Solid line, residual; dashed line, error on the objective function; dot-dashed line, value of the energy gain.

the other hand, the error $e$ can achieve the same level of convergence (with different rates) for all of the three cases. Therefore, for the present computations, we stop the convergence procedure when $e=10^{-6}$. Finally, although it is not possible to demonstrate that the optimal solution is indeed the global optimal of the nonlinear problem, we have verified that changing the initial guess of the optimization process does not change the optimal solution.

\section{Results}

\subsection{Non-linear optimal perturbations}

The nonlinear optimization procedure described in the previous section has been applied to Couette flow with $R e=400$. Figure 2 shows the value of the optimal energy gain versus the target time for $E_{0}=0.01$ (black circles) computed using the domain D1. The optimal energy gain obtained by the corresponding linear optimization is provided in the same figure (white squares), for comparison. For $T>20$, the nonlinear optimal energy gain is remarkably (up to 30 times) larger than the corresponding linear one. In particular, the energy gain grows in time until $T=90$, unlike the linear case in which a decay is obtained for $T>40$. The energy saturation for $T \geqslant 90$ is due to 


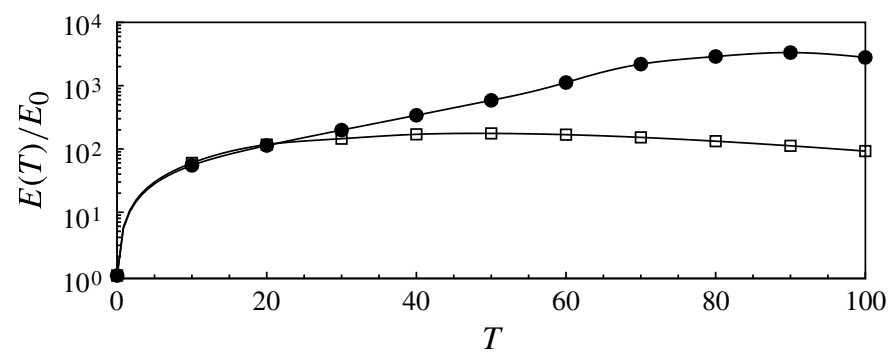

FIGURE 2. Optimal energy gain versus target time $T$ for $E_{0}=0.01$. White squares, linear optimization; black circles, nonlinear optimization.

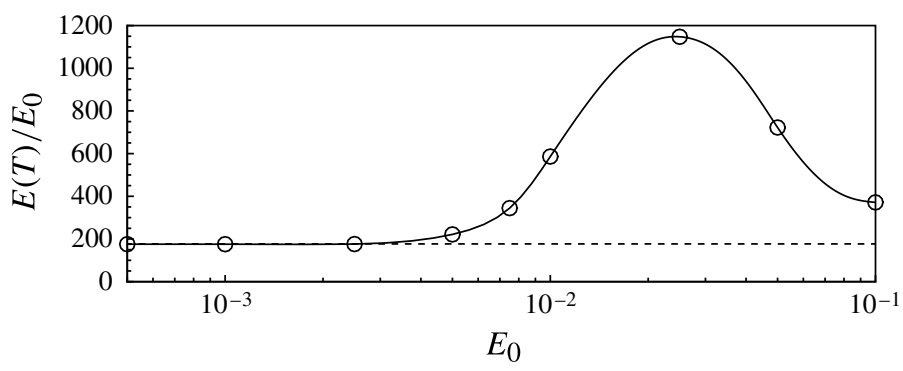

FIGURE 3. Optimal energy gain at target time $T=50$ for different values of the initial energy $E_{0}$. The dashed line represents the linear optimization result.

nonlinear effects, which do not allow an infinite growth of the perturbation energy in a domain of limited size. In fact, when the perturbations have filled the computational domain, the energy cannot grow further due to a spreading of the perturbation in space (for a comparison, see the case of the boundary layer in Cherubini et al. 2011). The influence of the parameter $E_{0}$ on the value of the optimal energy gain is shown in figure 3 , for $T=50$. The energy gain is approximately constant for small initial energies, its value being very close to that obtained by linear optimization, represented by the dashed line. For $E_{0}>0.0025$, the energy gain is found to deviate from the linear value, strongly increasing up to $E_{0} \approx 0.025$. The deviation from the linear behaviour appears to be smooth, unlike that found in Pringle et al. (2012), probably due to the low Reynolds number and short target time. When $E_{0}>0.025$, the energy gain remains larger than the linear one, but it is found to decrease, due to a saturation of the final energy value, $E(T)$, induced by nonlinear effects which are dominant for these large values of $E_{0}$. Therefore, it appears that a threshold exists for the value of the initial energy (target time) at a given target time (initial energy), from which non-negligible modifications are observed in the optimal energy with respect to the linear case; this threshold, which depends on both $E_{0}$ and $T$, will hereafter be called the nonlinearity threshold. Crossing such a threshold also yields large modifications in the shape of the optimal perturbations. This is clearly shown in figure 4, which provides the optimal initial perturbations obtained for $T=50$, for four values of the initial energy, $E_{0}$. For the lowest one, $E_{0}=0.001$ (figure $4 a$ ), the perturbation is similar to that obtained by the linear optimization; it is characterized by elongated vortices aligned with the $x$ direction. Notice that the patches of streamwise 

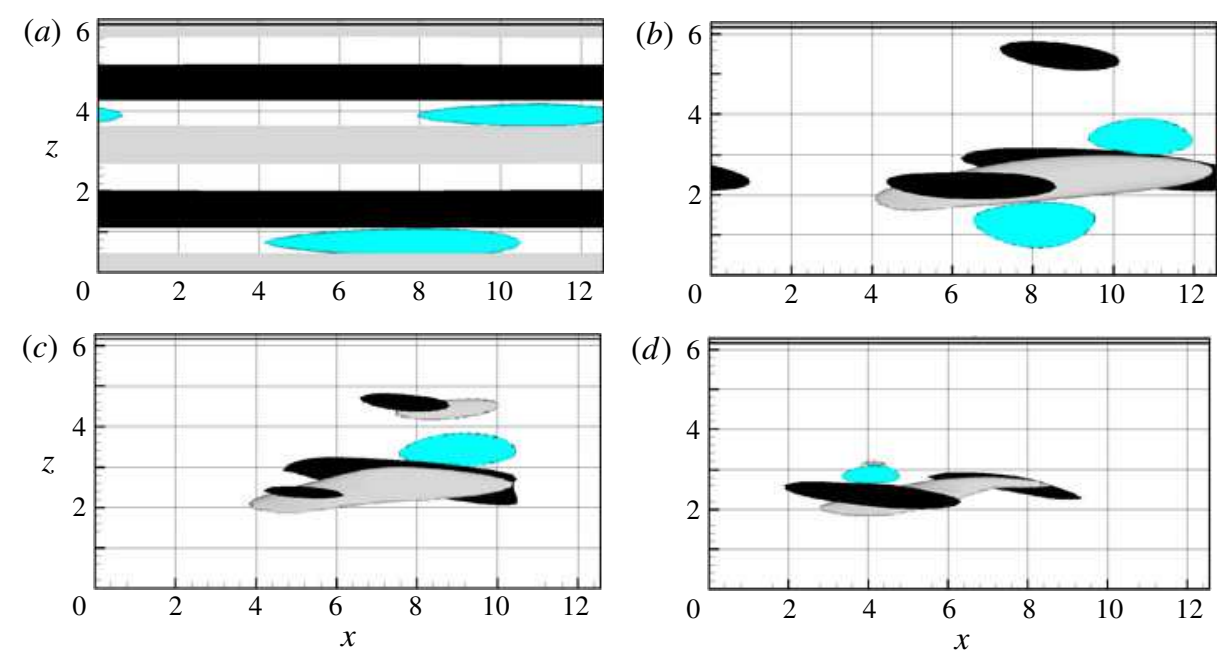

FIGURE 4. (Colour online) Initial perturbations obtained by the nonlinear optimization for target time $T=50$ : iso-surfaces of the optimal perturbations (grey, blue online, for the negative streamwise component of the velocity; black and pale grey for negative and positive streamwise vorticity, respectively) with initial energy $(a) E_{0}=0.001,(b) E_{0}=0.005$, (c) $E_{0}=0.01$, and $(d) E_{0}=0.05$. The velocity and vorticity perturbations have been normalized with respect to their maximum values. The surfaces represent the normalized values $\bar{u}^{\prime}=-0.085, \bar{\omega}_{x}^{\prime}= \pm 0.06$, which correspond to $(a) u^{\prime}=-0.00019, \omega_{x}^{\prime}= \pm 0.018$, (b) $u^{\prime}=-0.011, \omega_{x}^{\prime}= \pm 0.09,(c) u^{\prime}=-0.023, \omega_{x}^{\prime}= \pm 0.18$, and $(d) u^{\prime}=-0.06, \omega_{x}^{\prime}= \pm 0.5$.

perturbations, which can be due to weak nonlinear effects or to numerical noise, are very small in amplitude $\left(u^{\prime}=-0.00019\right)$. For $E_{0} \geqslant 0.005$, the structure of the optimal perturbation changes remarkably, being characterized by localized vortices, inclined and alternating with respect to the $x$ direction, placed around patches of large streamwise velocity perturbation. For larger initial energies, the optimal perturbation maintains a similar basic structure, although the vortices become more elongated and alternating in a different fashion; the perturbation also appears to be more localized in the spanwise direction and associated with larger values of the velocity amplitudes. Similar modifications are observed in the optimal perturbations when the target time is varied, as shown in figure 5. Being composed of streamwise vortices for $T<20$ (not shown), the optimal disturbance changes remarkably in shape around $T \approx 20$; for larger values of the target time slight modifications are observed, similar to those described above. We have thus observed that the nonlinear optimal perturbation has a basic structure which is found as soon as nonlinear effects become nonnegligible (namely, for values of the target time and initial energy larger than the nonlinearity threshold), and then changes slightly for a further increase of the initial energy or target time. Now it is important to verify that the optimal perturbation maintains this characteristic shape and size, varying the streamwise or spanwise domain length. Figure 6 shows the optimal disturbance obtained using the domain D2, for two different initial energies, $E_{0}=0.005$ and $E_{0}=0.1$. It appears that the optimal perturbation maintains its characteristic structure and that such a structure is replicated along $x$ and/or $z$ for increasing values of the initial energy (as also observed in a boundary layer: see Cherubini et al. 2011). This result could be anticipated since, due to the small target time, the basic structures cannot interact with each other. 

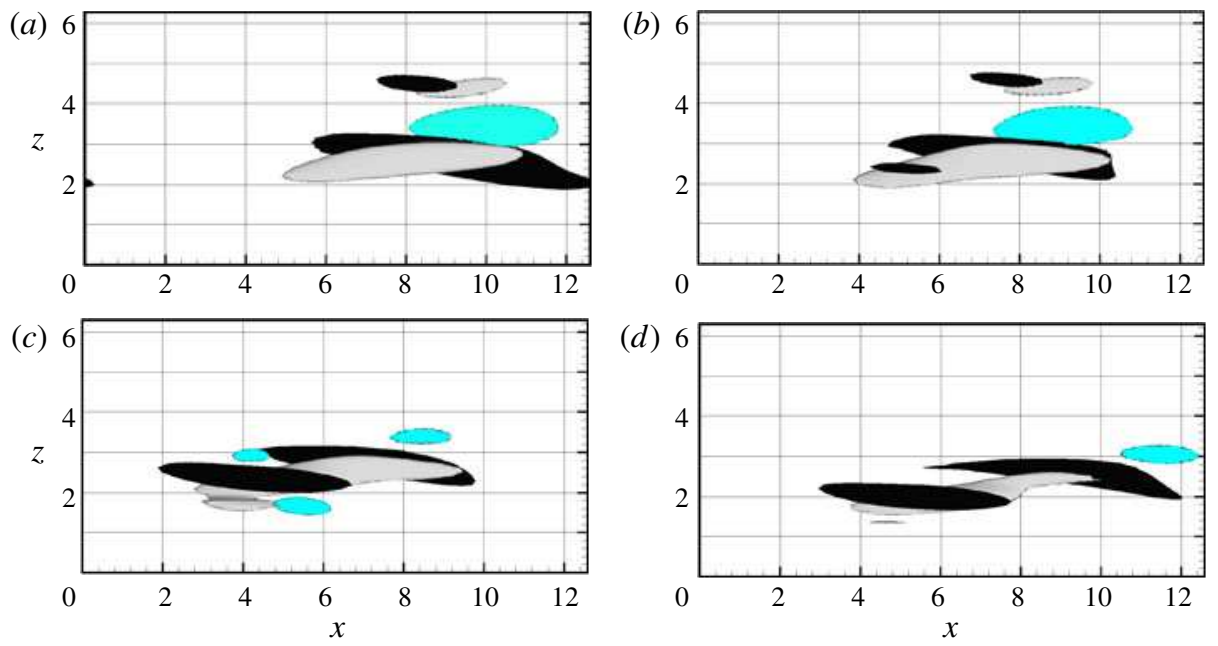

FIGURE 5. (Colour online) Initial perturbations obtained by the nonlinear optimization for initial energy $E_{0}=0.01$ : iso-surfaces of the optimal perturbations (grey, blue online, for the negative streamwise component of the velocity; black and pale grey for negative and positive streamwise vorticity, respectively) with target time (a) $T=30,(b) T=50,(c) T=70$, and (d) $T=90$. The surfaces with $u^{\prime}=-0.023, \omega_{x}^{\prime}= \pm 0.18$ are shown.
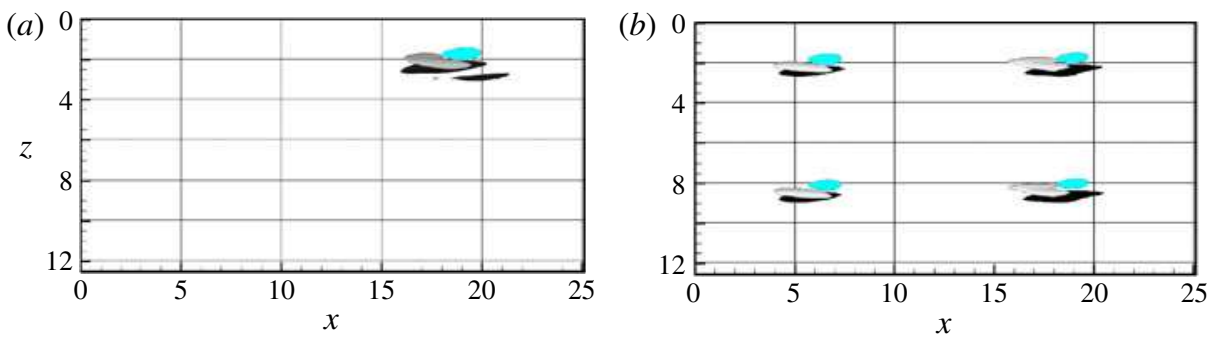

FIGURE 6. (Colour online) Initial perturbations obtained by the nonlinear optimization for $T=50$ in the computational domain D2: iso-surfaces of the optimal perturbations (grey, blue online, for the negative streamwise component of the velocity; black and pale grey for negative and positive streamwise vorticity, respectively) with initial energy (a) $E_{0}=0.005$ (surfaces for $u^{\prime}=-0.035, \omega_{x}^{\prime}= \pm 0.3$ ) and $(b) E_{0}=0.1$ (surfaces for $\left.u^{\prime}=-0.085, \omega_{x}^{\prime}= \pm 0.7\right)$.

The inclined vortices constituting the optimal disturbances are shown in figure 7, which provides four $x$-constant sections of the optimal initial perturbation obtained for $T=50$ and $E_{0}=0.01$. The main vortices are placed near $z \approx 2$, and a pair of weaker vortices are placed near $z \approx 5$. All of the vortices are inclined with respect to the base flow, in both the wall-normal and spanwise directions. The upstream tilting with respect to the wall-normal direction, which can be observed in figure 7, is linked to the Orr mechanism (see Schmid \& Henningson 2001), namely, a transient energy growth is produced when the mean flow tilts downstream the structures initially opposing the base flow. Such a tilting has been observed for linear optimal perturbations in shear flows (see Ehrenstein \& Gallaire 2008; 

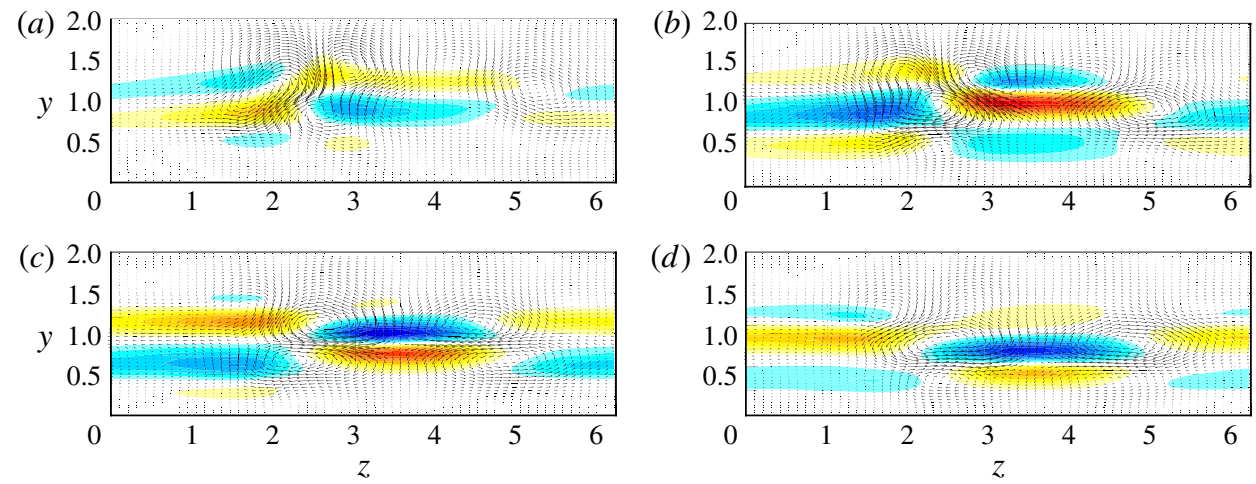

FIGURE 7. (Colour online) Contours and vectors of the velocity components of the initial optimal perturbation obtained for $T=50$ and $E_{0}=0.01$, on the planes $(a) x=4.5,(b) x=6.5$, (c) $x=8.5$, and $(d) x=10.5$. Shaded contours indicate the streamwise disturbance velocity (light, yellow online, for positive values; dark, blue online, for negative ones). Vectors represent the wall-normal and the spanwise disturbance velocity components.
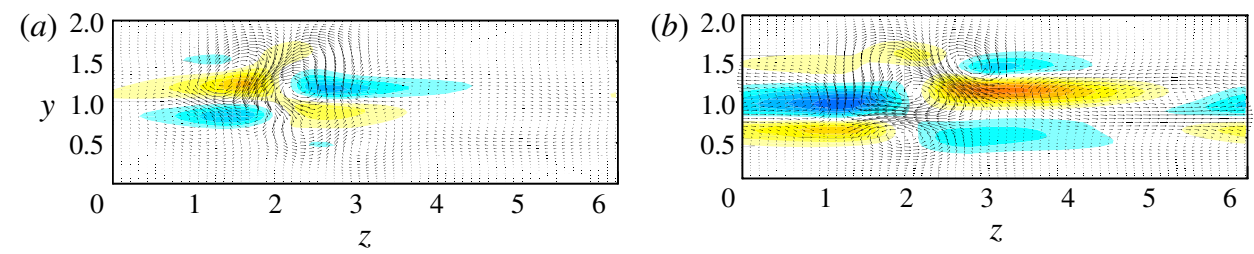

FIGURE 8. (Colour online) Contours and vectors of the velocity components of the initial optimal perturbation obtained for $T=90$, and $E_{0}=0.01$, on the planes $(a) x=4.5$ and (b) $x=8.5$. Shaded contours indicate the streamwise disturbance velocity (light, yellow online, for positive values; dark, blue online, for negative ones). Vectors represent the wallnormal and the spanwise disturbance velocity components.

Cherubini et al. 2010b). On the other hand, spanwise tilting is not observed in the linear optimal perturbation for the Couette flow, in which the optimal perturbations are characterized by elongated vortices perfectly aligned with the streamwise direction (Butler \& Farrell 1992). Moreover, one can notice the strong shear layers which are created between the zones of streamwise perturbation of opposite sign. Increasing the target time (or the initial energy), the structure of the disturbance remains similar, although the vortices are all localized in a smaller region in the spanwise direction, as shown in figure 8 for the nonlinear optimal at $T=90$ and $E_{0}=0.01$.

It is remarkable that the basic structure of such optimal perturbations is very similar to that found by means of nonlinear optimization in a boundary layer, consisting in alternating inclined vortices which lie on the flanks of a patch of large streamwise velocity perturbation. The following differences are recovered: (i) for Couette flow, nonlinear optimal perturbations are not symmetric with respect to the streamwise direction, whereas they are for boundary-layer flow; (ii) the velocity components have different relative magnitude. In fact, for Couette flow, the largest component of the velocity perturbation is the spanwise one $\left(w^{\prime}=0.037\right.$ for the case with $E_{0}=0.01$ and $T=50)$, which is comparable with the streamwise one $\left(u^{\prime}=0.033\right)$, and larger 


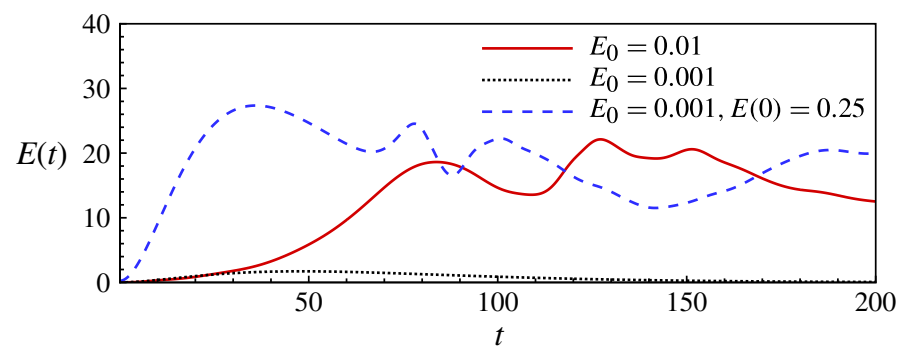

FIgURE 9. (Colour online) Energy versus time obtained by DNSs initialized using the optimal perturbations computed for $T=50$ for two different initial energies, $E_{0}=0.01$ (solid line) and $E_{0}=0.001$ (dotted line); the second perturbation has also been rescaled to $E(0)=0.25$ (dashed line).

than the wall-normal one $\left(v^{\prime}=0.027\right)$; whereas for boundary-layer flow, the largest component is the streamwise one.

For Couette flow considered here, the persistence of this basic structure at different values of the initial energy, domain sizes and target times indicates that the structure obtained by the nonlinear optimization, which maximizes the disturbance energy over a finite time, has an intrinsic fundamental importance. Moreover, the fact that very similar structures are recovered for boundary-layer flow indicates that such a basic structure might represent the most 'energetic' one at short target times for shear flows in general.

\subsection{The route to turbulence}

\subsubsection{Description of the transition process}

In this section, we study in detail the route to turbulence of the nonlinear optimal perturbations employing direct numerical simulation (DNS). For most of the computations, the optimal disturbance obtained for $T=50$ and $E_{0}=0.01$ has been used to initialize the flow field. Figure 9 provides the energy gain obtained by initializing the computations with the two optimal perturbations corresponding to $T=50, E_{0}=0.01$ (solid line) and $E_{0}=0.001$ (dotted line), respectively, the first perturbation being composed of inclined localized vortices, the second one by streamwise vortices having a very small streamwise modulation due to the presence of weak nonlinear interactions (the streamwise vortices deviate of \pm 0.02 in the spanwise direction with respect to their streamwise averaged $z=$ const. axis). One can observe that the first one leads the flow to turbulence, whereas the second one leads to relaminarization. This could be expected due to the lower initial energy of the second perturbation: thus, we have rescaled the initial energy of such an optimal perturbation until transition has been achieved. The rescaling has been applied after the optimization procedure using nine values of $E(0)$ in the range [0.0025, 0.25], and the resulting perturbation has been used to initialize the DNS. Notice that $E_{0}$ indicates the energy for which the nonlinear optimization has been performed, whereas $E(0)$ indicates the initial energy of a perturbation which has not been optimized for that particular initial energy. Thus, for such an initial disturbance composed of streamwise vortices (with a weak streamwise modulation), transition occurs for an initial energy $E(0) \approx 0.25$, whereas the nonlinear optimal disturbance composed of inclined localized vortices leads the flow to transition for a much lower initial energy, $E(0)=0.01$. This indicates that the streamwise inclination of the vortices is a crucial feature for 
transition to be induced effectively. Non-linear optimal perturbations computed for $E_{0}<0.01$ and $T=50$ do not lead to transition, although for such a target time the nonlinearity threshold is between $E_{0}=0.0025$ and $E_{0}=0.005$. This indicates that nonlinear effects are important also for the transient growth of perturbations below the laminar-turbulent boundary, as found by Pringle \& Kerswell (2010) for pipe flow.

The route to transition initiated by the nonlinear optimal perturbation at $E_{0}=$ 0.01 and $T=50$ is shown in figure 10, providing the surfaces of the negative streamwise component of the velocity perturbation, and of the negative and positive streamwise vorticity perturbations. At first, the inclined vortices transport the base flow momentum, inducing an increase of the streamwise component of the perturbation along them, creating localized zones of low- and high-momentum disturbance (see figure $10 b$ for $t=10$ ). At the same time, the streamwise vortices are stretched in the streamwise direction by the mean flow. Further base flow transport and stretching of the vortices induce the merging of the low- and high-momentum regions, creating elongated streaks having a spanwise modulation (see figure $10 \mathrm{c}$ for $t=30$ ); these are similar to the bent streaks observed by Monokrousos et al. (2010) in the early phases of transition initiated by their optimal perturbation, although in that case only a single streak is observed. Such streaks increase their strength due to the lift-up mechanism, and induce a higher inclination of the vortices through nonlinear coupling (figure $10 \mathrm{~d}$ for $t=50$ ). When the vortical structures are divided into smaller patches of vorticity and the streamwise streaks experience larger oscillations, the breakdown is initiated (figure $10 e, f$ for $t=70,90$ ). The flow maintains unsteady behaviour for some time; nevertheless, later we observe a partial decay of the disturbances, as shown by the energy curve in figure 11(a) (solid red line). In particular, at $t=210$ (figure 10g) one can observe inclined alternating vortices similar to those found at $t \approx 30$. However, this transient decay of the perturbation energy does not lead to relaminarization of the flow, but at larger times unsteady behaviour is recovered (figure 10h). This reorganization of the flow towards more coherent structures could be linked to a rapid passage in the vicinity of some exact coherent states; we will validate this conjecture in §3.2.3.

In order to analyse in detail the early phases of transition induced by the nonlinear optimal perturbations, it is interesting to determine the role of the nonlinear terms of the NS equations in the different stages of transition. Thus, the nonlinear terms (written in a conservative formulation) have been switched off one by one and nine modified direct numerical simulations have been performed, whose results are shown in figure 12. The perturbation energy time histories show that the most important term in the initial phase is $\left(w^{\prime} w^{\prime}\right)_{z}$, which is linked to the streamwise inclination of the initial vortices. In fact, when such a term is switched off, the initial inclined vortices are rapidly straightened by the mean flow, aligning themselves in the streamwise direction (see the inset of figure 12), as found for boundary-layer flow (see Cherubini et al. 2011). It appears that, although the streamwise vortices continue to induce streaks by means of the lift-up mechanisms, the absence of the nonlinear coupling term $\left(w^{\prime} w^{\prime}\right)_{z}$ hampers the sustainment of the vortices. In order to explain why such a term is crucial for the self-sustainment of the vortices, we could refer to the toy model proposed by Waleffe (1997), which mimics the basic mechanisms allowing a self-sustainment of turbulence. Such a model has also been used to explain the mechanisms leading to transition initial disturbances such as streamwise vortices or spanwise sinuous perturbations in the absence of streaks (see Cossu et al. 2011). The 
(a)

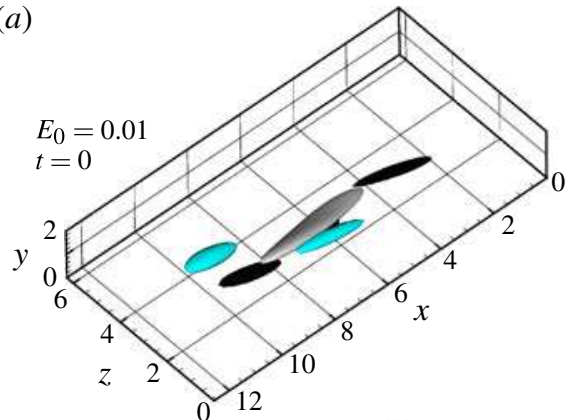

(c)

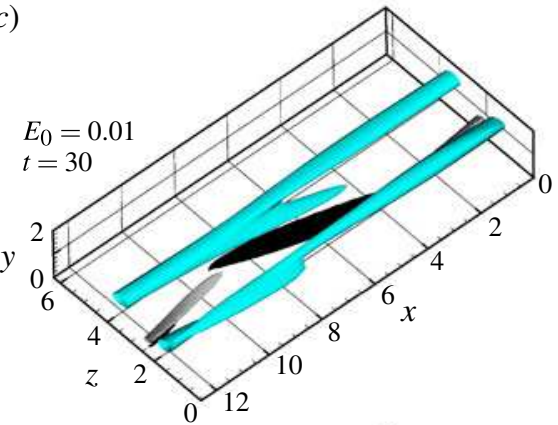

$(e)$

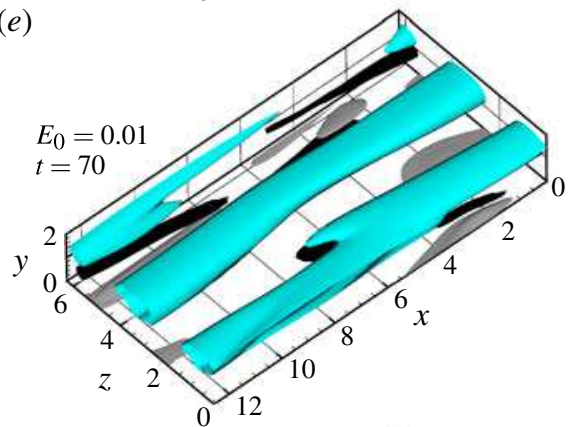

$(g)$

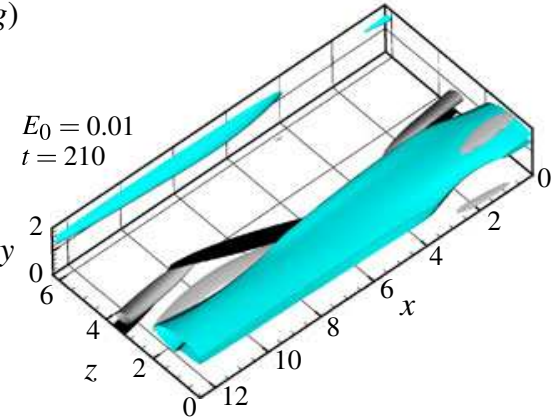

(b)

$(d)$
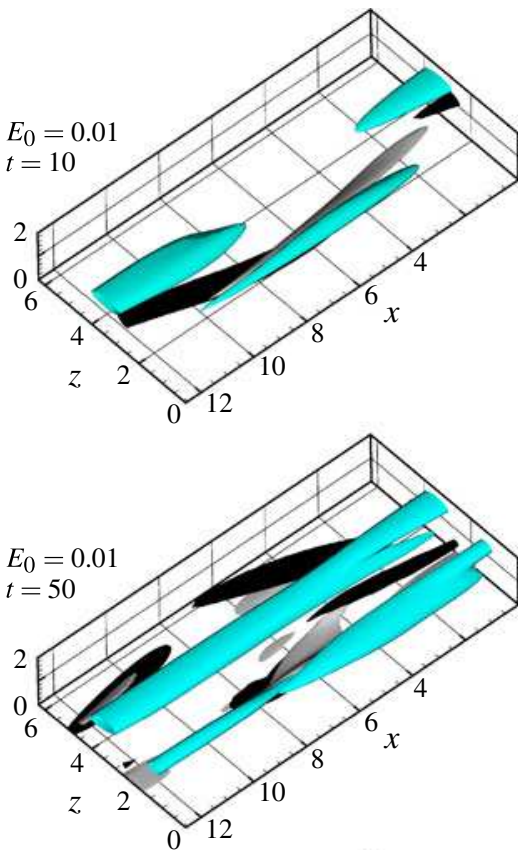

$(f)$

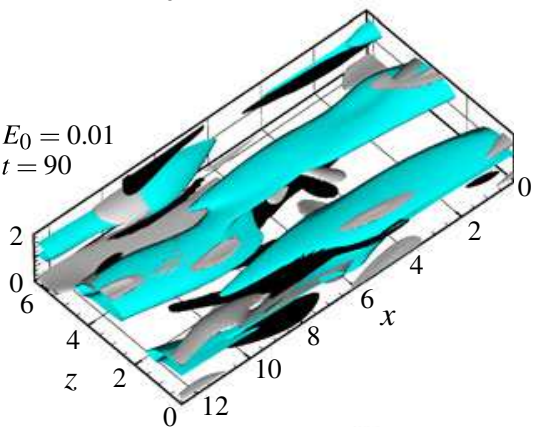

(h)

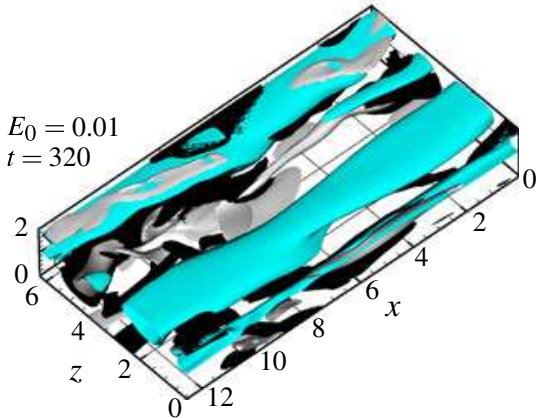

FIGURE 10. (Colour online) Snapshots of the time evolution of the nonlinear optimal perturbation obtained for $E_{0}=0.01$ and $T=50$ : iso-surfaces of the perturbations (grey, blue online, for the negative streamwise component of the velocity; black and pale grey for negative and positive streamwise vorticity, respectively) at (a) $t=0,(b) t=10,(c) t=30$, (d) $t=50,(e) t=70,(f) t=90,(g) t=210$ and $(h) t=320$. Surfaces for $(a, b) u^{\prime}=-0.015$, $\omega_{x}^{\prime}= \pm 0.5,(c) u^{\prime}=-0.025, \omega_{x}^{\prime}= \pm 0.75,(d-h) u^{\prime}=-0.035, \omega_{x}^{\prime}= \pm 0.75$. 

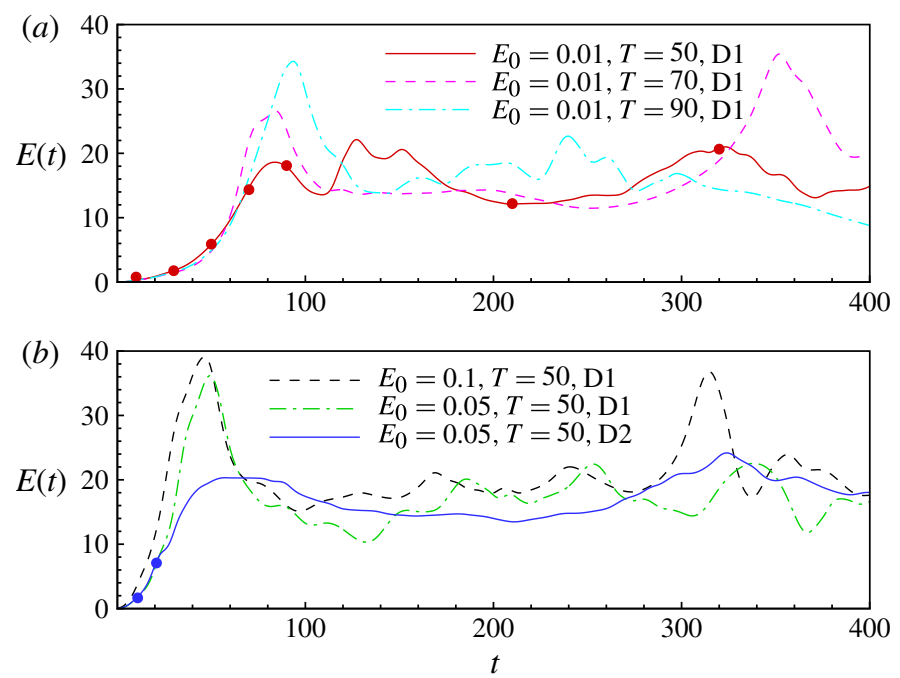

FIGURE 11. (Colour online) Energy versus time for six DNSs initialized by $(a)$ three optimal perturbations for $E_{0}=0.01$ with different target times, and $(b)$ three optimal perturbations for $T=50$ with two different initial energies and domains. The red and blue dots indicate the times at which the perturbations in figures 10 and 13 have been extracted, respectively.

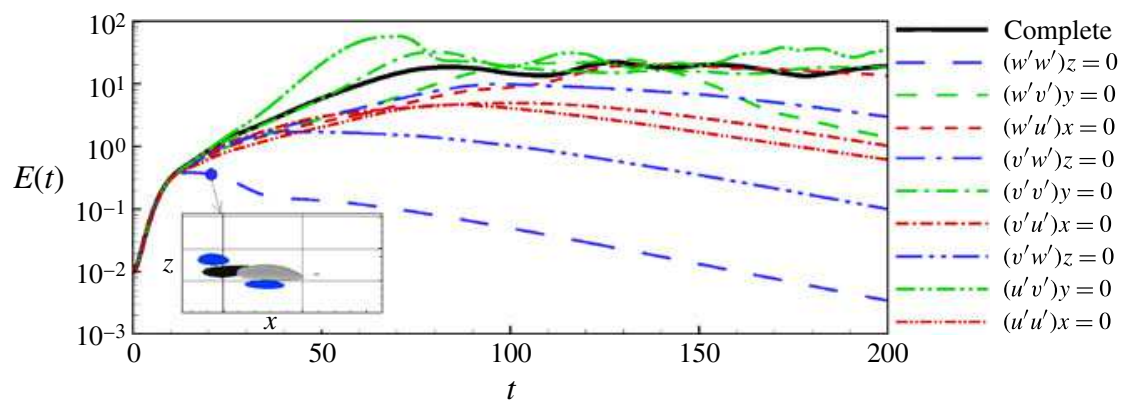

FIGURE 12. (Colour online) Energy versus time for a complete DNS (thick line) and nine DNSs obtained by switching off one by one the nonlinear terms indicated in the legend. The inset shows a snapshot of the evolution at $t=20$ of the nonlinear optimal perturbation with $T=50$ and $E_{0}=0.01$ for a DNS with the $\left(w^{\prime} w^{\prime}\right)_{z}$ term switched off.

model is as follows:

$$
\begin{aligned}
\mathrm{d} \tilde{u} / \mathrm{d} t & =-\left(k_{u}^{2} / R\right) \tilde{u}-\sigma_{w} \tilde{w}^{2}+\sigma_{u} m \tilde{v}, \\
\mathrm{~d} \tilde{v} / \mathrm{d} t & =-\left(k_{v}^{2} / R\right) \tilde{v}+\sigma_{v} \tilde{w}^{2}, \\
\mathrm{~d} \tilde{w} / \mathrm{d} t & =-\left(k_{w}^{2} / R\right) \tilde{w}+\left(\sigma_{w} \tilde{u}-\sigma_{m} m-\sigma_{v} \tilde{v}\right) \tilde{w}, \\
\mathrm{~d} m / \mathrm{d} t & =-\left(k_{m}^{2} / R\right) m-\sigma_{u} \tilde{u} \tilde{v}+\sigma_{m} \tilde{w}^{2}+k_{m}^{2} / R,
\end{aligned}
$$

where $\tilde{u}, \tilde{v}, \tilde{w}$ are the amplitudes of the streamwise streaks, the streamwise vortices, and the sinuous perturbations of the streaks, respectively and $m$ is the mean shear induced by these perturbations at Reynolds number $R$. Recently, Cossu et al. (2011) 
have shown that, in a Couette flow, transition can be obtained even if the amplitude of the initial streaks is negligible. Therefore, let us assume an initial condition composed essentially of vortices having wall-normal amplitude $\tilde{v}$, and spanwise amplitude $\tilde{w}$. The wall-normal amplitude $\tilde{v}$ is damped by the viscous term $-\left(k_{v}^{2} / R\right) \tilde{v}$, but it is also regenerated by the coupling term $\sigma_{v} \tilde{w}^{2}$. When the balance between these two terms is positive (for $\tilde{w}$ large enough), the vortices' amplitude grows in time, becoming a source of energy for the streaks (through the lift-up mechanism mimicked by the term $\sigma_{u} m \tilde{v}$ ) and then for the spanwise disturbances (through the coupling term $\left.\sigma_{w} \tilde{u} \tilde{w}\right)$. On the other hand, by neglecting the term $\sigma_{v} \tilde{w}^{2}$, the equation for $\tilde{v}$ would have only damping terms, so that the amplitude of the vortices would decay in time. Consequently, the equations for $\tilde{u}$ will also lose all of the source terms, and all of the perturbation components would decay in time. The toy model explains the importance in the early phases of transition of the coupling term $\left(w^{\prime} w^{\prime}\right)_{z}$, which allows the vortices to be sustained in time. This confirms the fundamental role of the initial inclination of the vortices in order to rapidly induce transition in the absence of initial streaks.

Another fundamental term in this transition scenario is the $\left(u^{\prime} w^{\prime}\right)_{z}$ term, which appears to be important for the self-sustainment of the vortices by the nonlinear coupling with the streaks. Similarly, one can see from the toy model that the $\sigma_{w} \tilde{u} \tilde{w}$ term is the only production term for the equation of the spanwise disturbance (recalling that the model holds for $\tilde{u}, \tilde{v}, \tilde{m}>0$ : see Waleffe 1997), so that switching it off induces a decay of the $\tilde{w}$ amplitude in time, hampering the establishment of a self-sustained cycle.

Other fundamental contributions for driving the perturbation to transition are linked to the $\left(u^{\prime} u^{\prime}\right)_{x}$ and $\left(u^{\prime} v^{\prime}\right)_{x}$ terms (see figure 12). Equivalent terms are not recovered in the toy model. In particular, our direct numerical simulations indicate that switching such terms off the streamwise perturbation straightens up, becoming quasi-streamwise independent at $t \approx 70$. Therefore, we can conclude that these terms contribute to sustaining the streamwise modulation of the streaks, which is very rapidly induced by the inclined vortices, allowing the flow to reach turbulence by a more rapid path than via the formation and successive secondary instability of streamwise streaks. These mechanisms cannot be recovered in the toy model, which is based on the latter scenario of transition; they will be discussed in further detail in \$3.2.2. Thus, the rapid path to transition induced by the nonlinear optimal perturbations relies on two main features: (i) the spanwise inclination of the initial vortices, which are tilted by the $\left(w^{\prime} w^{\prime}\right)_{z}$ term and sustained by the $\left(u^{\prime} w^{\prime}\right)_{z}$ term; (ii) the streamwise modulation of the streamwise disturbance, maintained by the terms $\left(u^{\prime} u^{\prime}\right)_{x}$ and $\left(u^{\prime} v^{\prime}\right)_{x}$, which allow the perturbation to skip the process of formation, saturation and secondary instability of the streamwise streaks. The contribution of the other nonlinear terms is small with respect to that of these four main terms; thus, we will not analyse their effect.

Optimal perturbations obtained for larger initial energies induce transition at even shorter times. Figure 13 shows the early phases of transition corresponding to the evolution of the nonlinear optimal perturbation obtained with initial energy $E_{0}=0.05$ for $T=50$. At $t=10$ (figure 13a), one can observe the lift-up mechanism producing patches of negative and positive streamwise disturbance, as well as the stretching of the localized inclined vortices by the mean flow, as already observed for lower initial energies. However, unlike the latter case, at $t=20$ (figure 13b) the flow is already becoming unsteady due to the local interaction of strong vortical structures. Such local interactions induce a very fast path to transition, resulting in a peak of the perturbation energy at $t \approx 50$ (the target time of the optimization), as shown in figure 11(b) by the dot-dashed curve $\left(E_{0}=0.05\right)$ and the dashed curve (for $\left.E_{0}=0.1\right)$. One can notice 
(a)

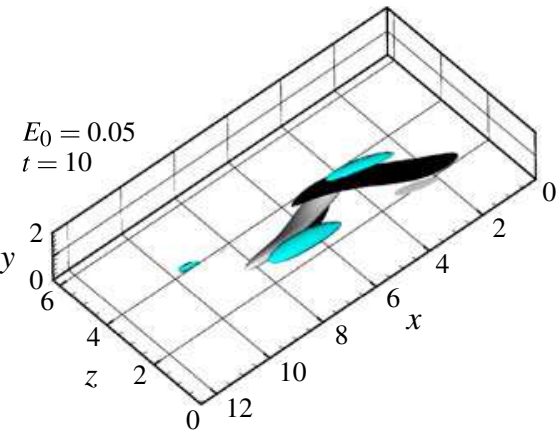

(b)

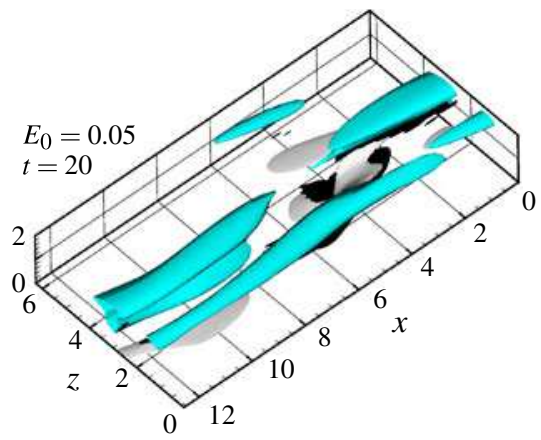

Figure 13. (Colour online) Snapshots of the perturbation at $(a) t=10$ and $(b) t=20$, for a DNS initialized by the initial optimal disturbance computed for $E_{0}=0.05, T=50$. Blue (grey) surfaces represent the negative streamwise component of the perturbation velocity $\left(u^{\prime}=-0.3\right)$; black and pale grey represent negative and positive streamwise vorticity perturbation $\left(w_{x}^{\prime}=1.2\right)$, respectively.

that the peak is transient; at larger times the perturbation energy attains lower values, which are typical of the turbulent state in Couette flows. However, such a peak is not only observed at short times, but it can also be achieved at larger times (see the black dashed curve for $E_{0}=0.1$ ). This behaviour lets us identify such energy peaks with bursting events of intense dissipation, as will be better explained in the next subsection. Note that, for the same initial energy and a larger domain (D2), the bursting events are not observed at short times, as shown by the blue solid curve in figure $11(b)$, the mechanism of transition being more similar to that observed for lower initial energies in the smaller domain. This is due to the fact that the optimal perturbation at $E_{0}=0.05$ for the domain D2 is composed of structures similar to those obtained at lower initial energies in the smaller domain, replicated in the streamwise and spanwise direction. Thus, for the same initial energy, the optimal disturbances in the two domains would have rather different shapes and amplitude (the one in D2 being characterized by lower amplitudes), thus not inducing bursting events at short times. It is also worth noticing that, if the DNS is initialized by the nonlinear optimal perturbation obtained for $E_{0}=0.01$ and $T=50$, but rescaled with an initial energy $E(0)=0.1$, the initial peak is characterized by smaller values of the energy, namely $E(t) \approx 28$ at $t=50$. This means that the occurrence of the bursting event is not only linked to the initial amplitude of the perturbation, but it is strongly dependent on its initial shape. In fact, the optimal disturbances at $E_{0}=0.05$ and $E_{0}=0.1$ are rather different with respect to that obtained for $E_{0}=0.01$ (see figure 4). This is further confirmed by observing in figure 11(a) that also the nonlinear optimal perturbations obtained for $E_{0}=0.01$ and $T=70,90$ (dashed and dot-dashed lines, respectively) induce bursting events at short times. Since their shape is very close to that obtained for larger initial energies and shorter target times, one can conclude that the initial shape of the perturbations, not their energy, is crucial to inducing a bursting event at short times.

\subsubsection{Comparison among the transition scenarios}

In order to compare the rapid path to transition of the nonlinear optimal disturbances with the classical scenario of transition for Couette flow, we measure the root mean square (r.m.s.) values of the velocity and vorticity components in direct numerical 
(a)

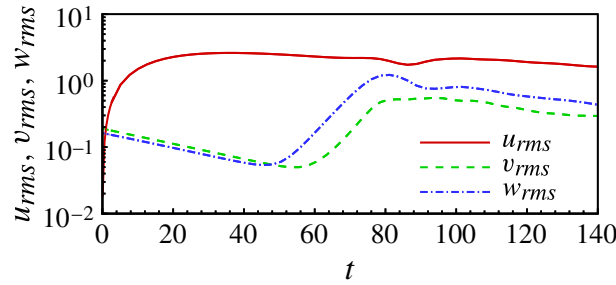

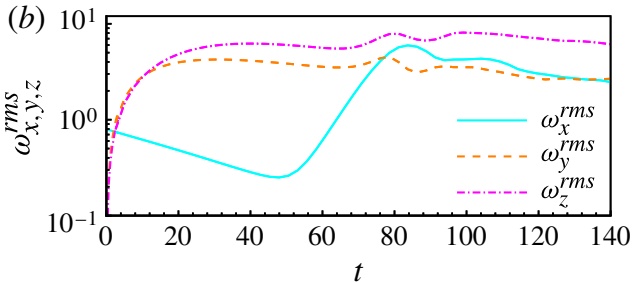

FIGURE 14. (Colour online) Root mean square values of the three components of $(a)$ velocity and $(b)$ vorticity versus time, extracted from a DNS initialized by the nonlinear optimal perturbations with $E_{0}=0.001$, which has been rescaled for the initial energy $E(0)=0.25$.

simulations initialized by different initial disturbances. The r.m.s. velocity values are defined as $u_{r m s}^{2}=\int_{V}(u-U)^{2} / U_{d}^{2} \mathrm{~d} V, v_{r m s}^{2}=\int_{V}(v)^{2} / U_{d}^{2} \mathrm{~d} V$, and $w_{r m s}^{2}=\int_{V}(w)^{2} / U_{d}^{2} \mathrm{~d} V$, where the reference velocity, $U_{d}$, is defined as the difference between the velocities of the two walls (see Cossu et al. 2011); a similar definition is employed for the r.m.s. vorticity. Figures 14-16 show the velocity and vorticity r.m.s. values for three direct numerical simulations initialized by the nonlinear optimal perturbations obtained with initial energies $E_{0}=0.001, E_{0}=0.01$ and $E_{0}=0.1$, respectively, with $T=50$. The initial energy of the first perturbation has been rescaled to $E(0)=0.25$ to achieve transition. The evolution of such a perturbation is provided in figure 14, which shows the classical transition scenario relying on the formation and secondary instability of the streaks. The initial streamwise vortices which constitute the (quasilinear) optimal perturbation transport the base flow momentum, inducing an increase of the streamwise component of the perturbation. On the other hand, the cross-stream components of the velocity slowly decay, until, at $t \approx 40$, a critical value of $u_{r m s}$ is reached which triggers the secondary instability of the saturated streaks. This induces the exponential growth of the spanwise and then wall-normal components of velocity, beginning the regeneration cycle theorized in Waleffe (1997). One can also note that the evolution of the streamwise vorticity follows the evolution of the spanwise component of the velocity. This can be easily explained by taking the curl of the vorticity, obtaining for the spanwise component:

$$
\nabla^{2} w=\partial \omega_{x} / \partial y-\partial \omega_{y} / \partial x
$$

$\omega_{y}$ and $\omega_{x}$ being the wall-normal and streamwise vorticity; considering that in the first phase of the transition process the flow structures do not present any streamwise variation, one can conclude that the streamwise vorticity is the only source term for $w$.

A very different picture is provided in figure 15 for the nonlinear optimal perturbation with initial energy $E_{0}=0.01$. All of the velocity components grow very rapidly (excluding a small initial decrease of $w$ for $t<5$ ), in particular the streamwise and the spanwise ones. The former clearly grows due to the lift-up effect induced by the initial vortices which transport the base flow momentum. The rapid growth of the spanwise component also appears to be driven by the streamwise vorticity (see (3.2), although this time the second term on the right-hand side is not zero), since both variables grow rapidly, peaking approximately at the same time instant. The initial growth of the streamwise vorticity confirms that this transition scenario relies on amplification mechanisms different from those observed in the previous case. In particular, in order to explain such an initial growth, we can consider the streamwise 
(a)

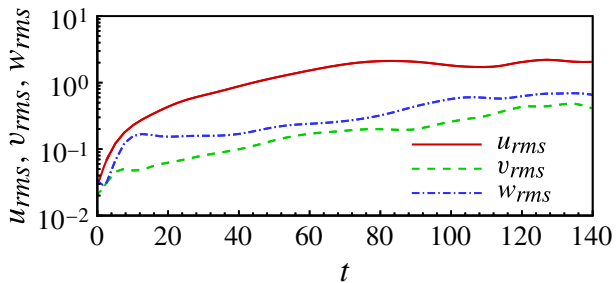

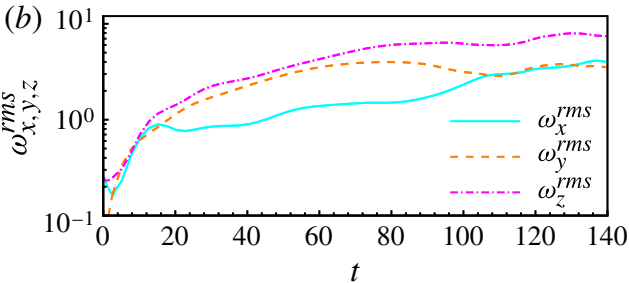

FIGURE 15. (Colour online) Root mean square values of the three components of $(a)$ velocity and $(b)$ vorticity versus time, extracted from a DNS initialized by the nonlinear optimal perturbations with $E_{0}=0.01$.

(a)

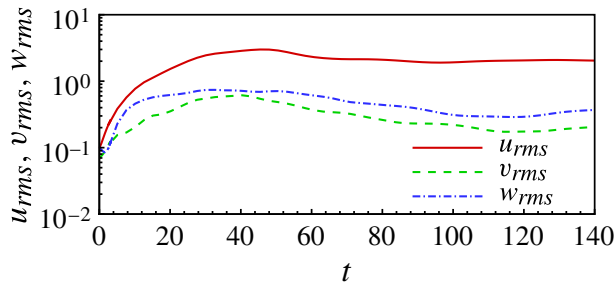

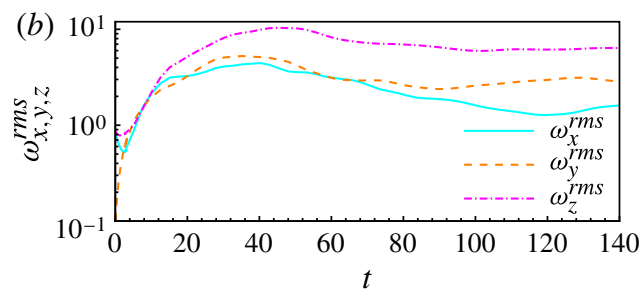

FIGURE 16. (Colour online) Root mean square values of the three components of $(a)$ velocity and $(b)$ vorticity versus time, extracted from a DNS initialized by the nonlinear optimal perturbations with $E_{0}=0.1$.

component of the vorticity transport equation, which reads

$$
\frac{\mathrm{D} \omega_{x}}{\mathrm{D} t}=\frac{\partial u}{\partial x} \omega_{x}+\frac{\partial u}{\partial y} \omega_{y}+\frac{\partial u}{\partial z} \omega_{z}+\frac{1}{R e} \nabla^{2} \omega_{x} .
$$

When we initialize the flow with streamwise vortices, the first term on the righthand side is zero, and the second and the third terms are small, since at $t=0$ the streamwise component of the perturbation is small. Thus, in order to induce an increase of the streamwise vorticity, the second and third terms of the equation should overtake the dissipation term, which happens only when the streamwise component of velocity (and the shear it induces) grows up to a critical value. On the other hand, nonlinear optimal perturbations are not streamwise invariant like the linear optimal ones, and they show large values of the streamwise component of velocity. Thus, the first term on the right-hand side of (3.3) is not zero, and the second and the third are larger than in the previous case. This allows the streamwise vorticity to overtake the viscous dissipation and to grow in time, self-sustaining the initial inclined vortices and causing an amplification of the streamwise disturbance along them by means of the lift-up effect. This confirms the fundamental role of the nonlinear terms $\left(u^{\prime} u^{\prime}\right)_{x}$, $\left(u^{\prime} v^{\prime}\right)_{x}$ in the early phases of transition, and explains the rapid path to transition of the nonlinear optimal disturbance. Summarizing, such a transition scenario relies on the following steps:

(a) initial amplification of the streamwise component of velocity due to the Orr and lift-up mechanisms;

(b) growth of the streamwise vorticity due to its coupling with the streamwise shear (3.3); 
(c) concurrent growth of the spanwise (and wall-normal) velocity components constituting the streamwise inclined vortices (3.2);

(d) sustainment and tilting of the vortices by feedback of the $\left(w^{\prime} w^{\prime}\right)_{z}$ and $\left(u^{\prime} w^{\prime}\right)_{z}$ nonlinear terms, inducing modulated streaks;

(e) establishment of modulated streaks and inclined vortices within the flow, finally leading to a regeneration cycle sustaining turbulence.

This transition scenario exploits the main fundamental features of the nonlinear optimal disturbances for shear flows (see also Pringle \& Kerswell (2010) and Cherubini et al. (2010b) for pipe flow and boundary-layer flow, respectively): (i) the large initial values of the streamwise velocity component; (ii) the streamwise dependence (and localization) of the disturbance; (iii) the presence of initial (inclined) streamwise vortices.

It is also worth noticing that this transition scenario is very different from the one discussed in Cossu et al. (2011), where it is shown that transition can be induced by an initial secondary sinuous perturbation in the presence of very weak primary streaks or vortices. This latter transition mechanism relies on the tilting of an initial wall-normal vorticity by the wall-normal shear, to allow an initial amplification of streamwise vorticity. Nevertheless, such a growth comes along with a decrease of the spanwise component of velocity, and a rapid increase of the wall-normal one, whereas in our case all of the velocity components grow together, the spanwise component more rapidly than the wall-normal one. This confirms the differences between the two transition scenarios, and gives evidence of the efficiency of nonlinear optimal perturbations at inducing transition in Couette flow.

Finally, note that, for a larger initial energy, $E_{0}=0.1$, at short times the velocity and vorticity components grow in a way similar to the case with $E_{0}=0.01$ (compare figures 15 and 16), although in this case the velocity and vorticity curves have a larger slope since unsteady behaviour is reached at a smaller time. Moreover, unlike the previous case, a saturation of the values of the velocity components is already reached at short times, due to the large growth of the perturbation energy in correspondence with the bursting event. This means that for these two cases the transition mechanism is similar, but for a larger initial energy it is characterized by a faster dynamics, due to the different shape and amplitude of the perturbations. The differences between these two cases will be explained in detail in $\S 3.2 .3$.

\subsubsection{Optimal perturbations in phase space}

Non-linear optimal perturbations computed here for different initial energies and target times have been found to be very efficient at inducing transition. In this section we want to study the interaction between the trajectories of such perturbations and the ECS populating phase space in the Couette flow. Figure 17 shows the projection of these trajectories onto the plane energy input $(I)$-dissipation rate $(D)$. The first three curves correspond to the optimal perturbations with initial energies $E_{0}=0.01$, $E_{0}=0.05$ and $E_{0}=0.1$, respectively, and $T=50$. The dots represent the points on each trajectory at the time instants $t=50, t=100$ and $t=150$, respectively, starting from the laminar solution at $D=I=1$. It appears that, for the lowest initial energy, the perturbation follows a short path trajectory towards the turbulent state (located at $I \approx D \approx 3$ : see Kawahara \& Kida 2001), and hereafter it will thus be called the short-path optimal perturbation; whereas for larger initial energies, the flow follows a trajectory characterized by large values of the dissipation rate $(D \approx 5)$. This behaviour corresponds to a bursting event, typical of shear flows, whose origin is still not clear, although it has very recently been linked, for Couette flow, to a homoclinic trajectory 


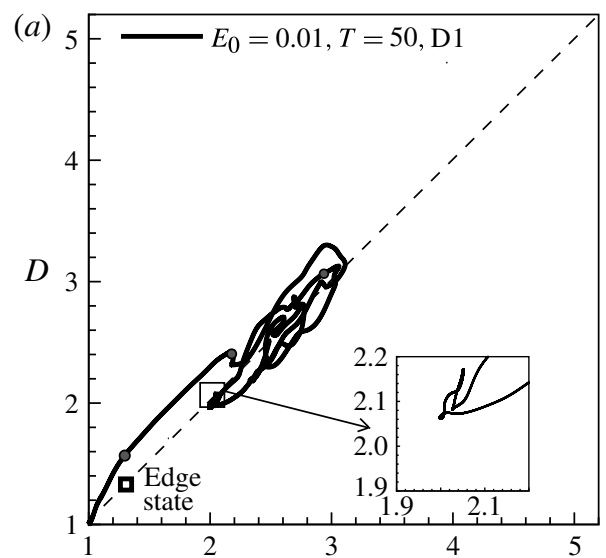

(b)

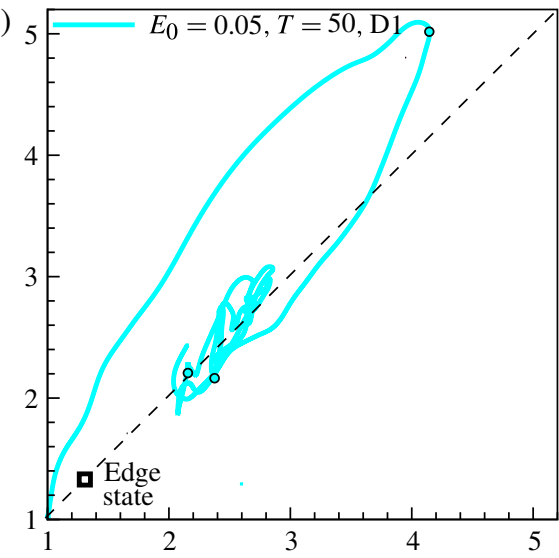

(c)

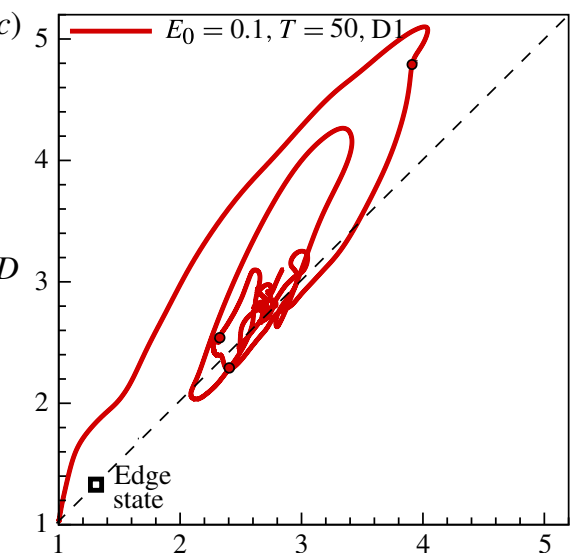

$(d)$

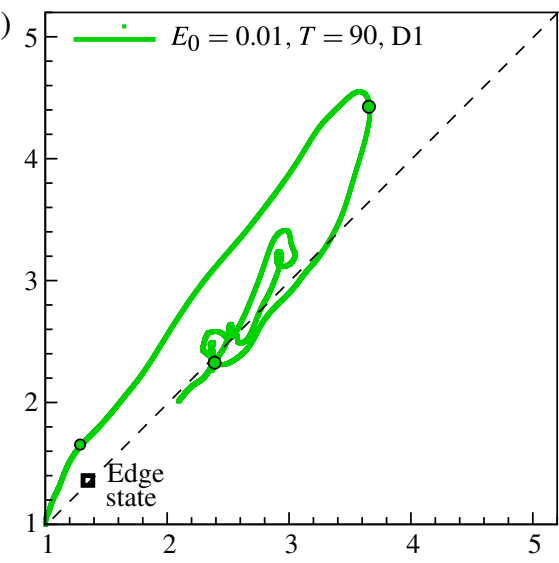

(e)
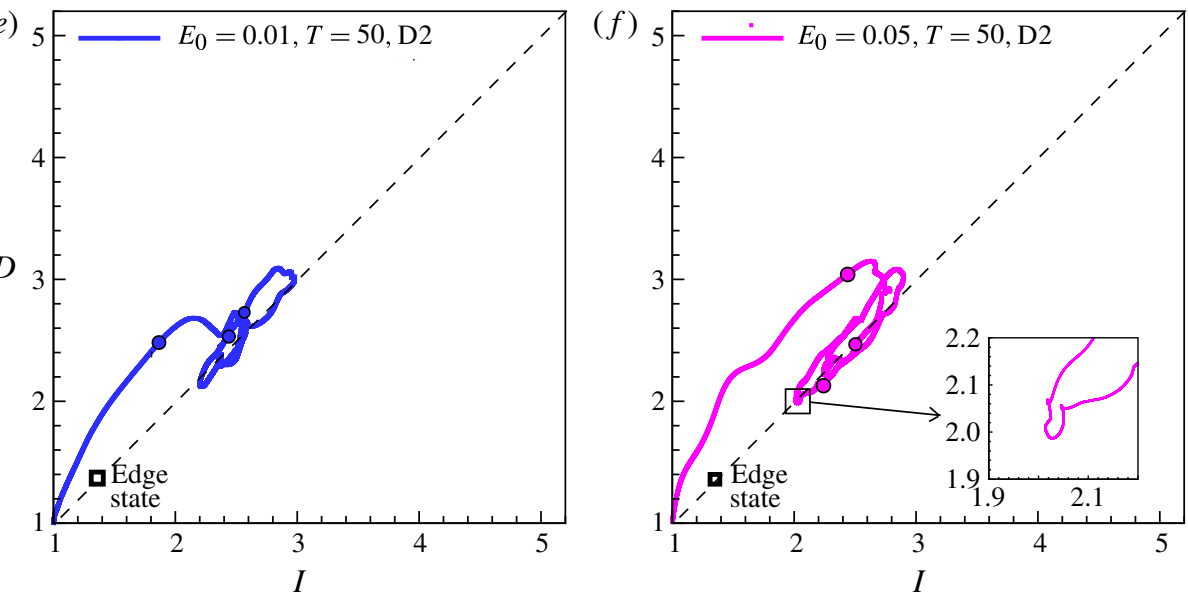

FIgURE 17. (Colour online) Trajectories in the two-dimensional phase space $I-D$ of the nonlinear optimal disturbances for the following initial energies, target times, and computational domains: (a) $E_{0}=0.01, T=50, \mathrm{D} 1$; (b) $E_{0}=0.05, T=50, \mathrm{D} 1$; (c) $E_{0}=0.1$, $T=50, \mathrm{D} 1 ;(d) E_{0}=0.01, T=90, \mathrm{D} 1 ;(e) E_{0}=0.01, T=50, \mathrm{D} 2 ;(f) E_{0}=0.05, T=50$, D2. All of the perturbations have been tracked up to $t=500$. The coloured dots indicate times $t=50,100,150$, increasing following the trajectory from the initial state close to $I, D \approx 1$. 
(a)

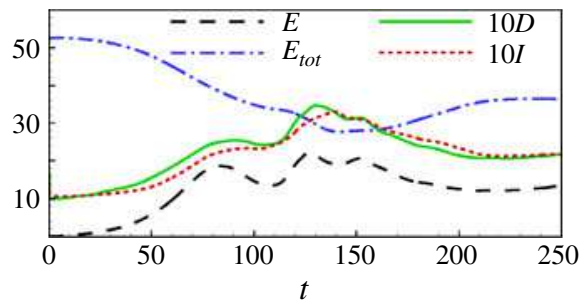

(b)

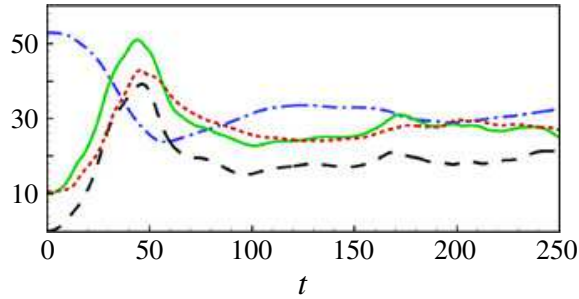

FIgURE 18. (Colour online) Perturbation energy (dashed lines), total energy (dot-dashed lines), dissipation rate (solid lines), and energy input (dotted lines) versus time for two DNSs initialized by the nonlinear optimal disturbances for the initial energies $(a) E_{0}=0.01$ and (b) $E_{0}=0.1$. In both cases the dissipation rate and the energy input have been multiplied by a factor of ten to make the comparison easier.

approaching an unstable periodic orbit characterized by weakly oscillating streaks (see van Veen \& Kawahara 2011). For the highest initial energy, two bursting events are observed, although the second event reaches values of $D$ slightly smaller than the first one. It is worth noticing that, at $t=50$, the short-path perturbation is still lying close to the laminar attractor $(D \approx 1.6)$, whereas at the same time the higher energy perturbations have reached the largest dissipation rate value, $D \approx 5$, demonstrating the very fast dynamics of the latter trajectory. Moreover, as can be verified by looking at figure 18, for all of the initial perturbations, the peak of the dissipation rate approximately corresponds to the peak of the perturbation energy (notice that in these figures the dissipation rate has been multiplied by a factor of 10 to allow easy comparison with the energy curve), explaining why, optimizing the energy gain, bursting events characterized by high dissipation values can be observed at short times. This behaviour can be explained by noticing that the only component of the base flow vorticity is $\Omega_{z}=-1$, so that

$$
\int_{V}|\omega|^{2} \mathrm{~d} V=\int_{V}\left[\omega_{x}^{\prime 2}+\omega_{y}^{\prime 2}+\left(\omega_{z}^{\prime}-1\right)^{2}\right] \mathrm{d} V .
$$

The increase of the dissipation rate in time can be observed when the sum of the squares of the vorticity perturbations exceeds the negative term $-2 \omega_{z}^{\prime}$. This is indeed obtained during the evolution of the nonlinear optimal perturbations, since the squares of the vorticity perturbations increase with the velocities, whereas the $-\int_{V} 2 \omega_{z}^{\prime} \mathrm{d} V$ term remains close to zero since the regions of positive and negative spanwise vorticity are found to approximately balance each other.

The fact that the dissipation rate initially increases following the growth of the perturbation energy may seem to conflict with the kinetic energy equation obtained by multiplying the NS equations $(2.1)$ by the instantaneous velocity vector $\boldsymbol{u}$. In fact, for Couette flow (with periodic boundary conditions) we obtain that the time derivative of the global kinetic energy, $E_{t o t}=\int_{V}\left[u^{2}+v^{2}+w^{2}\right] \mathrm{d} V$, is equal to the balance between the rate at which the viscous forces work on the walls (the energy input $I$ ) and the rate of loss of mechanical energy due to dissipation (the dissipation rate $D$ ), namely $\dot{E}_{t o t}=I-D$. Thus, when the dissipation rate is larger than the energy input (the trajectory passing in the region above the dashed bisector in figure 17), the global energy must decrease. Indeed, figure 18 shows that when $D>I(D<I)$ the global energy decreases (increases). Moreover, it appears that the evolution of the dissipation rate is very similar to the evolution of the perturbation energy. Therefore, 
it may happen that while the global energy is decreasing, the perturbation energy is increasing. This is due to the term $2 u^{\prime} U$, which is large and negative due to the growth of the streaks, usually characterized by an opposite sign with respect to the underlying base flow. Such a term can balance the perturbation energy growth, inducing a decrease of the global energy and a further increase of the dissipation rate with respect to the energy input.

We have observed that nonlinear optimal perturbations with a rather high initial energy can induce bursting at short times, since a large growth of the perturbation energy appears to be linked to a large growth of the dissipation rate. However, bursting events are observed also for lower values of the initial energy, for instance $E_{0}=0.01$, when a larger target time $(T \geqslant 70)$ is chosen for the optimization, as shown by the green curve in figure $17(d)$. Comparing the optimal perturbation for $T=70,90$ and $E_{0}=0.01$ (figure $5 c, d$ ), with that for $T=50$ and $E_{0}=0.05$ (figure $4 d$ ), it appears that they present a similar shape, which is rather different from the short-path perturbation shown in figure $4(c)$. Thus, the occurrence of the bursting event appears to be linked to an initial perturbation of that particular shape, and not simply to a larger initial energy or amplitude of the disturbances. It is possible to confirm such a conjecture by looking at figure $17(e, f)$, showing the trajectories of the optimal initial perturbations computed for the same initial energies of figure $17(a, b)$, respectively, but for the larger domain D2. One can observe that, for the larger initial energy, the trajectories are strongly different in the two domains; in particular, bursting events are not observed in the larger domain. Therefore, bursting is not linked to the initial energy of the perturbations, but to their shape, since we have already observed that in a larger domain the optimal perturbation is composed of the same basic building block characterizing the short-path optimal disturbance, replicated in the streamwise and spanwise directions.

Thus, it is possible to conclude that we have found two classes of optimal perturbations characterized by rather different shapes and evolutions: the first one is the perturbation which most effectively induces a bursting event, hereafter called the highly dissipative optimal perturbation; the second class directly leads towards turbulence, therefore it is called the short-path optimal perturbation. To better characterize the second class of optimal disturbances, we can observe that all of the trajectories shown in figure 17 are strongly deviated around $I \approx 1.4$, which is the energy input typical of the ECS tracked on the edge of chaos by Schneider et al. (2008), hereafter called edge state (ES). One can also observe that the trajectory followed by the nonlinear optimal with $E_{0}=0.01$ passes closer to such an equilibrium solution. In order to establish if the evolution of the short-path perturbations is influenced by the presence of the ES, we compute the state on the laminar-turbulent boundary for the present flow by following the tracking procedure proposed by Skufca et al. (2006), and compare it with the flow structures obtained at a short time by initializing the flow with a short-path disturbance. The ES is shown in figure 19(a) and is compared to a snapshot extracted at $t=80$ from the DNS initialized by the optimal disturbance with $E_{0}=0.01$ and $T=50$, shown in figure $19(b)$. The similarity of the two flow structures indicates that the flow is attracted by and spends some time in the vicinity of the edge state, before reaching the turbulent attractor. To further confirm such an observation, we have computed the correlation $C$ of the flow state with the ES, using the formula proposed by Kerswell \& Tutty (2007), namely

$$
C(t)=\max _{x_{0}, z_{0}}\left[\frac{\left\langle\boldsymbol{v}_{D N S}, \boldsymbol{v}_{E S}\right\rangle}{\left.\left.\sqrt{(}\left\langle\boldsymbol{v}_{D N S}, \boldsymbol{v}_{D N S}\right\rangle\right) \sqrt{(}\left\langle\boldsymbol{v}_{E S}, \boldsymbol{v}_{E S}\right\rangle\right)}\right],
$$



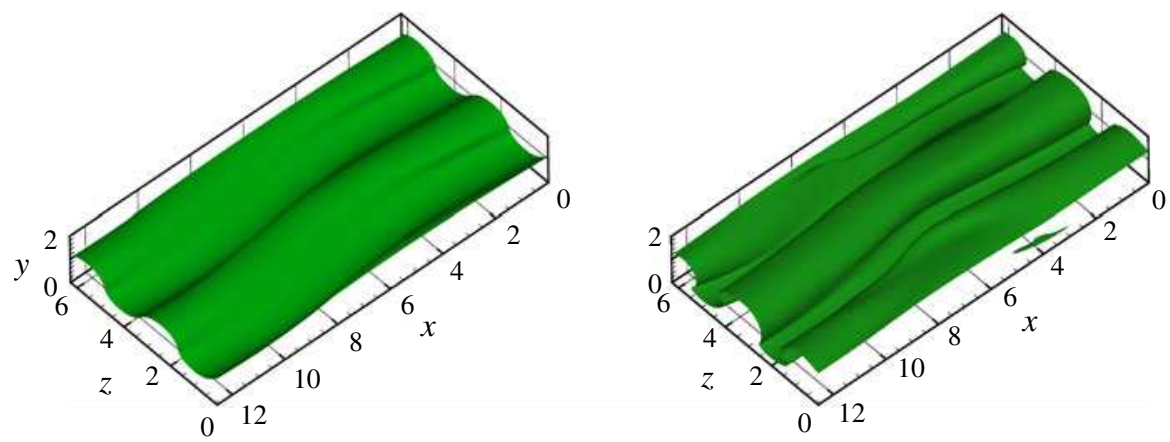

FIGURE 19. (Colour online) Null streamwise velocity contours for $(a)$ the equilibrium solution found by bisection, and $(b)$ a snapshot extracted at $t=80$ by the DNS initialized by the short-path perturbation.
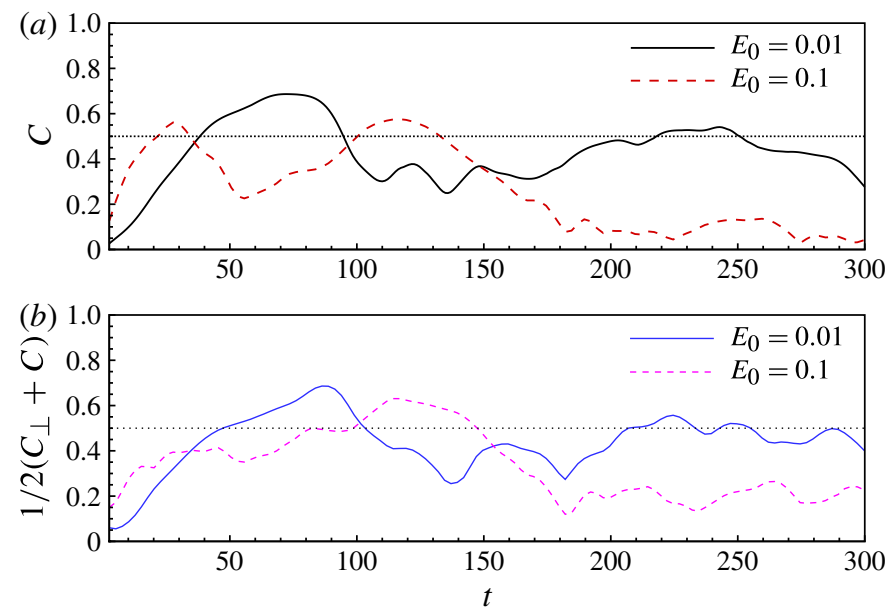

FIGURE 20. (Colour online) Correlations $(a) C$ and $(b) 1 / 2\left(C+C_{\perp}\right)$ between the equilibrium solution and the velocities found by DNS initialized with the nonlinear optimal perturbation for $T=50$ and for two different initial energies, $E_{0}=0.01$ (solid lines) and $E_{0}=0.1$ (dashed lines).

where $\langle\cdot, \cdot\rangle$ is the scalar product defined in (2.2), and the mean profile (in $x$ and $z$ ) of the ES $\left(\overline{\boldsymbol{u}}_{E S}\right)$ has been subtracted from the velocity field extracted by the DNS and from the equilibrium solution itself:

$$
\begin{gathered}
\boldsymbol{v}_{D N S}=\boldsymbol{u}_{D N S}\left(x+x_{0}, y, z+z_{0}\right)-\overline{\boldsymbol{u}}_{E S}, \\
\boldsymbol{v}_{E S}=\boldsymbol{u}_{E S}(x, y, z)-\overline{\boldsymbol{u}}_{E S} .
\end{gathered}
$$

Figure 20(a) shows that when the flow is initialized by the short-path disturbance (with $E_{0}=0.01$ and $T=50$ ), the correlation achieves rather high values (up to 0.7 ) for a large time interval $(C>0.5$ for $40 \lesssim t \lesssim 95)$, confirming that the perturbation spends some time near the edge of chaos before being deviated to turbulence. Perturbations of larger initial energy also move close to the ES at a small time, but they are rapidly deviated away from it (see the dashed line in figure 20a). This corresponds 

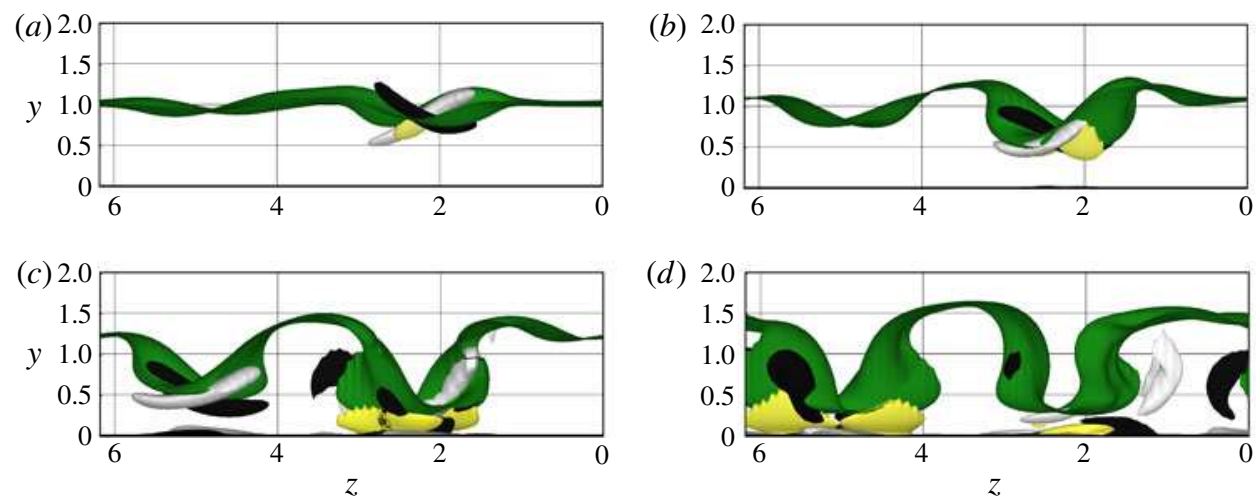

FIGURE 21. (Colour online) Null streamwise velocity contours (dark, green online), streamwise vorticity perturbation (black and pale grey for positive and negative values), and local dissipation rate (light, yellow online), extracted from a DNS initialized by the nonlinear optimal disturbance obtained for $E_{0}=0.01$ and $T=50$, at times $(a) t=10$ (surfaces for $\omega_{x}= \pm 0.6, \epsilon=5$ ), (b) $t=30$ (surfaces for $\omega_{x}= \pm 0.6, \epsilon=12$ ), $(c) t=50$ and $(d) t=70$ (surfaces for $\omega_{x}= \pm 0.6, \epsilon=16$ ).

to the different behaviour we have observed in phase space, indicating that the shortpath perturbation is able to bring the flow easily on the laminar-turbulent boundary, whereas the highly dissipative ones are the perturbations which effectively lead to a bursting event. To validate such a result, following Kerswell \& Tutty (2007) we have also computed the cross-stream correlation, $C_{\perp}$, defined by (3.5) using the crossstream velocity vector $\boldsymbol{u}=(0, v, w)^{\mathrm{T}}$. The curves in figure $20(b)$, showing the quantity $1 / 2\left(C+C_{\perp}\right)$, are similar to those previously analysed (although the correlation values for the highly dissipative perturbation are lower at short times), confirming the above analysis.

The different behaviour of the two classes of perturbations can be further investigated by analysing the evolution in time of the null streamwise velocity contours, which represent the streaks, and the streamwise vorticity contours. Figure 21 shows that when the flow is initialized by the short-path disturbance, the alternating streamwise vortices start to bend the null- $u$ surface (dark, green online surface in figure $21 a$ for $t=10$ ) which is at first localized close to $y \approx 1$. Such a bending increases in time, curving the surface towards the walls (figure $21 b$ for $t=30$ ). At $t=50$ (figure $21 c$ ), the null- $u$ surface is characterized by two hills of similar size, similar to the ES, and two pairs of vortices lying in the valleys below the hills. These vortices are stretched and wrapped, producing vortices on the flanks of a pair of bent streaks (figure $21 d$ for $t=70$ ). From that time on, the regeneration cycle of Waleffe (1997) is established and turbulence is sustained. By inspecting the surfaces of the local dissipation rate, $\epsilon$ (light, yellow online), we can observe that at short times the most dissipative zones are placed in the region of interaction of the two initial vortices; when time increases, such dissipative regions are displaced towards the lower wall.

For the highly dissipative nonlinear optimal perturbation with $E_{0}=0.05$, the early steps towards transition are similar to the previous case (see figure $22 a$ for $t=10$ ) although the vortices and the bending they induce are stronger. On the other hand, one can observe that the regions of high local dissipation rate are very rapidly transported towards the wall (compare the snapshot in figure $21 c$, extracted at $t=50$, with that of figure $22 b$, extracted at $t=20$ ), and the vortices begin to be wrapped already 


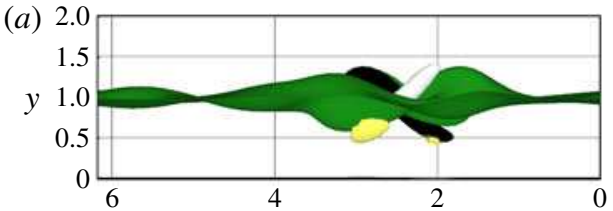

(b) 2.0

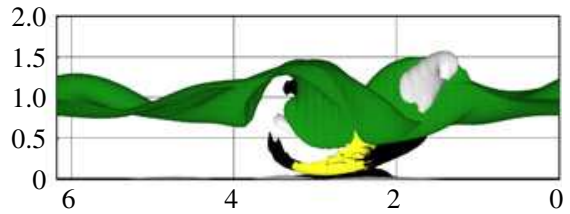

(c)

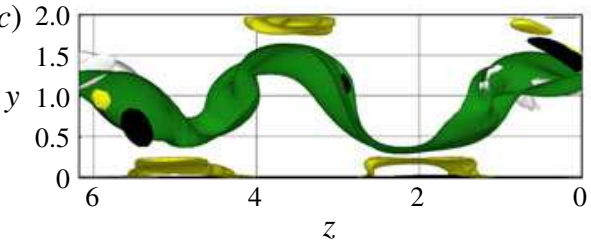

(d)

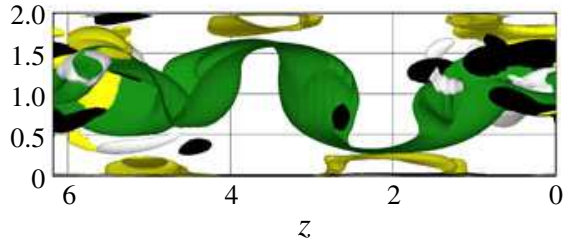

FIGURE 22. (Colour online) Null streamwise velocity contours (dark, green online), streamwise vorticity perturbation (black and pale grey for positive and negative values), and local dissipation rate (light, yellow online), extracted from a DNS initialized by the highly dissipative perturbation at $E_{0}=0.05$ and $T=50$, at times $(a) t=10$ (surfaces for $\omega_{x}= \pm 1.5$, $\epsilon=8$ ), (b) $t=20$ (surfaces for $\omega_{x}= \pm 1.5, \epsilon=16$ ), $(c) t=140$ (surfaces for $\omega_{x}= \pm 0.7$, $\epsilon=8$ ), and $(d) t=160$ (surfaces for $\omega_{x}= \pm 0.7, \epsilon=8$ ).

at a small time, leading to unsteady behaviour without spending much time close to the ES. However, at larger times, a transient decrease of the perturbation energy is observed (see the dot-dashed curve in figure $11 b$ at $t \approx 120$ ), which coincides with a transient reorganization of the flow into more coherent structures. Figure $22(c)$ provides the streamwise streaks and vortices for $t=140$, showing structures which are very similar to those found in the previous case at $t=50$, although this time the local dissipation rate is localized in quasi-streamwise invariant regions on the crests and valleys of the streaks. This similarity may seem unexpected since the trajectories in phase space do not go back to the edge state (indicated by a black square in figure 17). On the other hand, all of the trajectories are found to linger in a zone of phase space with $I \approx D \approx 2$ (see the insets in figure $17 a, f$ ). Such values of the dissipation rate and of the energy input are typical of the gentle unstable periodic orbit (UPO) found by Kawahara \& Kida (2001), which is very similar to the ES, but slightly oscillating in time. The passage in the vicinity of such a periodic orbit could explain the reorganization of the flow into coherent streaky and vortical structures, followed by a successive deviation towards the turbulent attractor. This links the results found here to the recent findings of van Veen \& Kawahara (2011), who conjecture that the bursting events are linked to the existence of a homoclinic orbit approaching the UPO. During the bursting event on such a homoclinic orbit, they observe streamwise vortices on the crests and valleys of the streaks, which are not usually observed in the regeneration cycle; thus they conclude that such vortices are typical flow structures of bursting since they have significant energy dissipation. Furthermore, we have observed vortices inducing high dissipation regions on the crests and valleys of the streaks since the early phases of transition, for both short-path and highly dissipative disturbances, although for the former disturbance the dissipation rate is localized only on the lower wall and is characterized by lower values with respect to the latter. Such differences can be better observed in figure 23, providing the local dissipation rate and the null streamwise velocity contours extracted at $t=60$ (figure 23a,b) and $t=80$ (figure 23c,d) from two direct numerical simulations initialized by two nonlinear optimal perturbations obtained with $E_{0}=0.01$ 

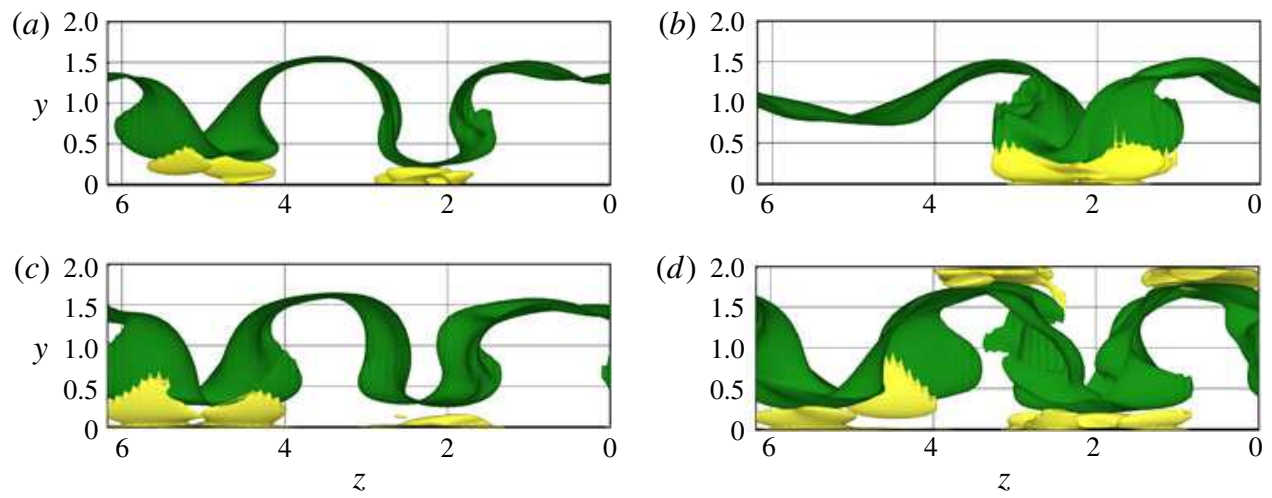

FIGURE 23. (Colour online) Null streamwise velocity contours (dark, green online surfaces) and local dissipation rate (light, yellow online surfaces for $\epsilon=16$ ), extracted at $(a, b) t=60$ and $(c, d) t=80$ from two DNSs initialized by the nonlinear optimal disturbances obtained for the initial energy $E_{0}=0.01$ and target times $(a, c) T=50$ (short-path) and $(b, d) T=90$ (highly dissipative).

and two different target times $T=50$ (figure 23a,c) and $T=90$ (figure 23b,d). The two perturbations have the same initial energies (and similar amplitudes), but rather different shapes. In particular, the short-path one (the one with $T=50$ ) has a larger spanwise extent than the highly dissipative one, which is more spanwise localized but is more extended in the streamwise direction (compare figure $5 b, d$ ). The result of this initial difference is that, at short times, the local dissipation rate is transported towards the wall at two spanwise locations for the former case, and at one location in the latter (compare figure 23a,b). A larger localized peak in the second case induces a larger bending of the streaks, which in turn leads to the creation of strong peaks of dissipation on both walls (see figure $23 d$ ), whereas in the first case the dissipation remains confined on the lower wall (see figure 23c). The discrepancy in the evolution of these two initial perturbations can be explained by recalling that, in the case of short-path perturbation, the flow is strongly attracted by the ES, which deviates its trajectory towards turbulence by inducing a regeneration cycle of Waleffe (1997) type. On the other hand, the highly dissipative perturbations appear to: (i) be rapidly repelled by the ES; (ii) reach high values of the dissipation; (iii) be attracted by the UPO; (iv) go back towards high values of the dissipation (figure 17c) or directly towards turbulence (figure 17b). A passage in the vicinity of the unstable periodic orbit is characterized by the organized structures shown in figure $22(c, d)$, which are very close to the structures found in the last stages of the homoclinic orbit by van Veen \& Kawahara (2011). Notice that this happens during the dissipative phase of the trajectory, and not during the initial energy-increasing phase, when the trajectory passes close to the ES. Indeed, the first stages of the route towards such a periodic solution are different from that found in van Veen \& Kawahara (2011), since we start from a perturbed laminar solution, and not from the UPO itself. Moreover, the trajectories are found to pass in the vicinity of an equilibrium solution which does not exist in the smaller domain used in that work, but which has a structure very similar to the UPO. Thus, it is possible to conjecture that, in a domain in which both the equilibrium solution of Schneider et al. (2008) and the periodic orbit of Kawahara \& Kida (2001) exist, a bursting event can be obtained not only as a homoclinic orbit 
approaching the UPO, but also as a heteroclinic orbit between the ES and the UPO. The highly dissipative perturbation thus appears to be able to pass close to such a heteroclinic orbit, starting from the laminar solution.

\section{Summary}

We have used a variational procedure to identify nonlinear optimal disturbances in a Couette flow, defined as those initial perturbations yielding the largest energy growth at a short target time $T$, for given Reynolds number $R e$ and initial energy $E_{0}$. For values of $E_{0}$ and $T$ larger than a nonlinearity threshold, the nonlinear optimal disturbances have been found to be characterized by a basic structure, composed of inclined streamwise vortices on the flanks of localized regions of low and high momentum. Such a basic structure closely recalls that found in other shear flows, such as boundary-layer flow, indicating that this structure may be the most 'energetic' one at short target times for this class of flows. Although the structure remains the same, when the values of $E_{0}$ and $T$ are increased beyond the nonlinearity threshold, some small modifications of the spanwise localization of the vortices are observed. This induces speculation about two different paths to transition, which are studied in detail by means of direct numerical simulations. In particular, direct numerical simulations have shown that optimal disturbances obtained for large $E_{0}$ and $T$ induce bursting events at short times, whereas for lower values of these parameters the flow is directly attracted towards the turbulent state. For this reason, the optimal disturbances have been classified into two classes, the highly dissipative and short-path optimal disturbances. Due to the short-time optimization, both classes lead the flow to turbulence, skipping the phases of streak formation and secondary instability typical of the classical transition scenario for Couette flow. In particular, the nonlinear transition scenario relies on the following steps:

(a) initial amplification of the streamwise component of velocity due to the Orr and lift-up mechanisms;

(b) growth of the streamwise vorticity due to the coupling with the streamwise shear induced by the finite-amplitude streamwise component of the perturbation;

(c) concurrent growth of the spanwise (and wall-normal) velocity components constituting the streamwise inclined vortices;

(d) sustainment and tilting of the vortices by feedback of the $\left(w^{\prime} w^{\prime}\right)_{z}$ and $\left(u^{\prime} w^{\prime}\right)_{z}$ nonlinear terms, inducing modulated streaks;

(e) establishment of modulated streaks and inclined vortices which sustain turbulence.

This transition scenario exploits three main features of the nonlinear optimal disturbances in shear flows: (i) the large initial values of the streamwise velocity component; (ii) the streamwise dependence of the disturbance; (iii) the presence of initial (inclined) streamwise vortices.

Although the mechanisms leading to transition are the same, the two classes of perturbations are found to behave very differently when looking at their trajectory on the $I-D$ (energy input-dissipation rate) projection of phase space. In particular, the short-path perturbations are found to spend a considerable amount of time in the vicinity of the edge state (which coincides with the equilibrium solution obtained by Schneider et al. (2008)) in the first phase of their route to transition. Also the highly dissipative optimal disturbances pass close to the edge state, but they are found to be rapidly repelled away from it, leading the flow to high values of the dissipation rate $(D \approx 5)$. After this dissipation peak, the trajectories do not lead towards the turbulent 
attractor, but they spend some time in the vicinity of the unstable periodic orbit (UPO) of Kawahara \& Kida (2001). This links our results to the recent findings of van Veen \& Kawahara (2011), who conjecture that bursting events are due to passages along homoclinic orbits approaching the UPO. Along these orbits streamwise vortices are observed on the crests and valleys of the streaks, having significant dissipation close to the walls, which are usually not recovered in the regeneration cycle. These structures are very similar to the ones observed here when the laminar solution is perturbed by the highly dissipative perturbations. Thus we conjecture that a bursting event can also be obtained as a heteroclinic orbit between the equilibrium solution on the edge and the UPO. The highly dissipative perturbations are able to rapidly join such a heteroclinic orbit starting from the laminar solution, whereas the short-path ones are strongly attracted towards the edge state, directly reaching the turbulent state. This different behaviour appears to be due to the different localization of the disturbances, inducing stronger local peaks of the dissipation rate in the former with respect to the latter case.

These results indicate a new rapid path to transition in a Couette flow, mostly relying on nonlinear mechanisms, which reproduces the typical features of subcritical transition to turbulence in shear flows, such as the spatial and temporal intermittency characterizing the bursting phenomenon.

\section{REFERENCES}

Butler, K. M. \& FARrell, B. F. 1992 Three-dimensional optimal perturbations in viscous shear flow. Phys. Fluids A 4, 1637-1650.

Cherubini, S., De Palma, P., Robinet, J.-C. \& Bottaro, A. $2010 a$ Rapid path to transition via nonlinear localized optimal perturbations. Phys. Rev. E 82, 066302.

Cherubini, S., De Palma, P., Robinet, J. C. \& Bottaro, A. 2011 The minimal seed of turbulent transition in a boundary layer. J. Fluid Mech 689, 221-253.

Cherubini, S., Robinet, J.-C., Bottaro, A. \& De Palma, P. $2010 b$ Optimal wave packets in a boundary layer and initial phases of a turbulent spot. J. Fluid Mech. 656, 231-259.

Cossu, C., Brandt, L., Bagheri, S. \& Henningson, D. S. 2011 Secondary threshold amplitudes for sinuous streak breakdown. Phys. Fluids 23, 074103.

Eckhardt, B., Schneider, T. M., Hof, B. \& WesterweEl, J. 2007 Turbulence transition of pipe flow. Annu. Rev. Fluid Mech. 39, 447-468.

Ehrenstein, U. \& Gallaire, F. 2008 Global low-frequency oscillations in a separating boundary-layer flow. J. Fluid Mech. 614, 315-327.

Faisst, H. \& Eckhardt, B. 2003 Travelling waves in pipe flow. Phys. Rev. Lett. 91, 224502.

Gibson, J. F., Halcrow, J. \& CVitanović, P. 2009 Equilibrium and traveling-wave solutions of plane Couette flow. J. Fluid Mech. 638, 243.

Hof, B., van Doorne, C. W. H., Westerweel, J., Nieuwstadt, F. T. M., Faisst, H., Eckhardt, B., Wedin, H., Kerswell, R. R. \& WalefFe, F. 2004 Experimental observation of nonlinear traveling waves in turbulent pipe flow. Science 305, 1594-1598.

Itano, T. \& Tон, S. 2001 The dynamics of bursting process in wall turbulence. J. Phys. Soc. Japan 70, 703-716.

Jimenez, J., Kawahara, G., Simens, M. P. \& Nagata, M. 2005 Characterization of near-wall turbulence in terms of equilibrium and 'bursting' solutions. Phys. Fluids 17, 015105.

KawaharA, G. \& KIDA, S. 2001 Periodic motion embedded in plane Couette turbulence: regeneration cycle and burst. J. Fluid Mech. 449, 291-300.

Kerswell, R. R. \& Tutty, O. R. 2007 Recurrence of travelling waves in transitional pipe flow. J. Fluid Mech. 584, 69-102.

Kline, S. J., Reynolds, W. C., Schraub, F. A. \& Rundstadler, P. W. 1967 The structure of turbulent boundary layers. J. Fluid Mech. 30, 741-773. 
Monokrousos, A., Akervik, E., Brandt, L. \& Henningson, D. S. 2010 Global three-dimensional optimal disturbances in the Blasius boundary-layer flow using time-steppers. J. Fluid Mech. 650, 181-214.

Monokrousos, A., Bottaro, A., Brandt, L., Di Vita, A. \& Henningson, D. S. 2011 Non-equilibrium thermodynamics and the optimal path to turbulence in shear flows. Phys. Rev. Lett. 106, 134502.

NAGATA, M. 1990 Three-dimensional finite-amplitude solutions in plane Couette flow. J. Fluid Mech. 217, 519-527.

Nagata, M. 1997 Three-dimensional traveling-wave solutions in plane Couette flow. Phys. Rev. E 55, 2023.

Pringle, C. C. T. \& Kerswell, R. R. 2010 Using nonlinear transient growth to construct the minimal seed for shear flow turbulence. Phys. Rev. Lett. 105, 154502.

Pringle, C. C. T., Willis, A. P. \& Kerswell, R. R. 2012 Minimal seeds for shear flow turbulence: using nonlinear transient growth to touch the edge of chaos. J. Fluid. Mech. 702, 415-443.

Robinson, S. K. 1991 Coherent motions in the turbulent boundary layer. Annu. Rev. Fluid Mech. 23, 601 .

Schmid, P. \& Henningson, D. 2001 Stability and Transition in Shear Flows. Springer.

Schneider, T. M., Gibson, J. F., Lagha, M., De Lillo, F. \& Eckhardt, B. 2008 Laminar-turbulent boundary in plane Couette flow. Phys. Rev. E 78, 037301.

SkufCA, J. D., Yorke, J. A. \& ECKhardt, B. 2006 Edge of chaos in a parallel shear flow. Phys. Rev. Lett. 96, 174101.

Van Veen, L. \& Kawahara, G. 2011 Homoclinic tangle on the edge of shear turbulence. Phys. Rev. Lett. 107, 114501.

VERZICCO, R. \& ORLANDI, P. 1996 A finite-difference scheme for the three-dimensional incompressible flows in cylindrical coordinates. J. Comput. Phys. 123 (2), 402-414.

WAlefFe, F. 1997 On a self-sustaining process in shear flows. Phys. Fluids 9, 883-901.

WALefFe, F. 1998 Three-dimensional states in plane shear flow. Phys. Rev. Lett. 81, 4140-4143.

WALEFFE, F. 2003 Homotopy of exact coherent structures in plane shear flows. Phys. Fluids 15, $1517-1534$.

WAng, J., Gibson, J. \& WALEFFe, F. 2007 Lower branch coherent states in shear flows: transition and control. Phys. Rev. Lett. 98, 20450.

Wedin, H. \& KERSWELl, R. R. 2004 Exact coherent structures in pipe flow: travelling wave solutions. J. Fluid Mech. 508, 333-371.

Zuccher, S., Luchini, P. \& BottARo, A. 2004 Algebraic growth in a Blasius boundary layer: optimal and robust control by mean suction in the nonlinear regime. Eur. J. Mech. B (Fluids) 513, 135-160. 